TITLE:

\title{
Rarefied gas flow around a sharp edge induced by a temperature field
}

AUTHOR(S):

Taguchi, Satoshi; Aoki, Kazuo

CITATION:

Taguchi, Satoshi ...[et al]. Rarefied gas flow around a sharp edge

induced by a temperature field. JOURNAL OF FLUID MECHANICS 2012, 694: 191-224

ISSUE DATE:

2012-01-17

URL:

http://hdl.handle.net/2433/171947

RIGHT:

(C) Cambridge University Press 2012 


\section{Journal of Fluid Mechanics}

http://journals.cambridge.org/FLM

Additional services for Journal of Fluid Mechanics:

Journal of

Fluid Mechanics

Email alerts: $\underline{\text { Click here }}$

Subscriptions: Click here

Commercial reprints: Click here

Terms of use : $\underline{\text { Click here }}$

\section{Rarefied gas flow around a sharp edge induced by a temperature field}

Satoshi Taguchi and Kazuo Aoki

Journal of Fluid Mechanics / Volume 694 / March 2012, pp 191 - 224

DOI: 10.1017/jfm.2011.536, Published online: 17 January 2012

Link to this article: http://journals.cambridge.org/abstract S0022112011005362

How to cite this article:

Satoshi Taguchi and Kazuo Aoki (2012). Rarefied gas flow around a sharp edge induced by a temperature field. Journal of Fluid Mechanics,694, pp 191-224 doi:10.1017/jfm.2011.536

Request Permissions : $\underline{\text { Click here }}$ 


\title{
Rarefied gas flow around a sharp edge induced by a temperature field
}

\author{
Satoshi Taguchi ${ }^{1} \dagger$ and Kazuo Aoki ${ }^{2}$ \\ ${ }^{1}$ Department of Mechanical Engineering and Intelligent Systems, University of Electro-Communications, \\ Chofu, Tokyo 182-8585, Japan \\ 2 Department of Mechanical Engineering and Science and Advanced Research Institute of Fluid Science \\ and Engineering, Graduate School of Engineering, Kyoto University, Kyoto 606-8501, Japan
}

(Received 14 July 2011; revised 10 October 2011; accepted 4 December 2011; first published online 17 January 2012)

A rarefied gas flow thermally induced around a heated (or cooled) flat plate, contained in a vessel, is considered in two different situations: (i) both sides of the plate are simultaneously and uniformly heated (or cooled); and (ii) only one side of the plate is uniformly heated. The former is known as the thermal edge flow and the latter, typically observed in the Crookes radiometer, may be called the radiometric flow. The steady behaviour of the gas induced in the container is investigated on the basis of the Bhatnagar-Gross-Krook (BGK) model of the Boltzmann equation and the diffuse reflection boundary condition by means of an accurate finite-difference method. The flow features are clarified for a wide range of the Knudsen number, with a particular emphasis placed on the structural similarity between the two flows. The limiting behaviour of the flow as the Knudsen number tends to zero (and thus the system approaches the continuum limit) is investigated for both flows. The detailed structure of the normal stress on the plate as well as the cause of the radiometric force (the force acting on the plate from the hotter to the colder side) is also clarified for the present infinitely thin plate.

Key words: kinetic theory, microfluidics, non-continuum effects

\section{Introduction}

When a flat plate, one side of which is kept at a higher temperature than the other, is placed in a rarefied gas that is otherwise at rest, a flow is induced and a force is exerted on the plate from the hotter side to the colder one. This is the well-known radiometric phenomenon and can be observed in the famous Crookes radiometer. It received significant attention in the early days of kinetic theory (Reynolds 1876; Maxwell 1879; Einstein 1924; Kennard 1938; Loeb 1961). Recently, there has been a revival of interest in this phenomenon (Scandurra, Iacopetti \& Colona 2007; Selden et al. 2009a,b; Cornella et al. 2011; Gimelshein et al. 2011a,b) due to its relation to various applications in micro-technologies (see e.g. Wadsworth \& Muntz 1996; Ota, Nakao \& Sakamoto 2001 and the references in Selden et al. 2009b). Despite its long

$\dagger$ Email address for correspondence: taguchi@mce.uec.ac.jp 
history, however, the mechanism of the flow and the force has not yet been fully understood, particularly when the molecular mean free path is small compared with the size of the plate (i.e. the near continuum regime).

In a previous study (Taguchi \& Aoki 2011), we have investigated a radiometric flow (i.e. a flow in a radiometer) numerically on the basis of the Bhatnagar-Gross-Krook (BGK) model (Bhatnagar, Gross \& Krook 1954; Welander 1954) of the Boltzmann equation. It was found that there is a structural similarity between the radiometric flow and the thermal edge flow, the latter of which is induced around the sharp edge of a uniformly heated (or cooled) plate (with both sides kept at the same temperature). This is a newer type of rarefied gas flow induced by the thermal effect, discovered by Aoki, Sone \& Masukawa (1995) by means of a numerical simulation using the direct simulation Monte Carlo (DSMC) method (Bird 1976, 1994), and later confirmed experimentally by Sone \& Yoshimoto (1997). Hence, it is important to consider not only the radiometric flow but also the thermal edge flow simultaneously to understand the mechanism of the radiometric flow and force. However, due to the limited space, no information on the thermal edge flow is given in our previous paper, nor a comparison between the two flows. In this paper, therefore, we carry out a more systematic study on the radiometric flow and the thermal edge flow, by extending the previous numerical analysis, and clarify the similarity as well as the difference embedded in the two flows.

Before ending this section, we briefly comment on the thermally induced flow from a general point of view. According to the asymptotic theory of the Boltzmann equation for small Knudsen numbers (Sone 1969, 1971, 1991; Sone et al. 1996; Sone 2002, 2007), the (steady) thermally induced flows are categorized into the following three types: (i) thermal creep flow (Kennard 1938; Sone 1966; Ohwada, Sone \& Aoki 1989a; Sharipov \& Seleznev 1998; Garcia \& Siewert 2009); (ii) thermal stress slip flow (Sone \& Tanaka 1980; Sone 2000); and (iii) nonlinear thermal stress flow (Galkin, Kogan \& Fridlender 1971; Kogan, Galkin \& Fridlender 1976; Sone 2000). The magnitude of the thermal creep flow is proportional to the mean free path, that of the thermal stress slip flow to the mean free path squared and that of the nonlinear thermal stress flow to the mean free path. The last flow is negligible when the temperature variation of the gas is small. On the other hand, the asymptotic theory is based on the assumption that the shape of the boundary is smooth. Therefore, if this condition is violated, another type of flow is possible. The thermal edge flow and the radiometric flow considered in this study are typical examples of such flows, to which the asymptotic theory does not apply even if the Knudsen number is small. Therefore, we require further investigations to clarify the individual properties of these flows.

The remainder of the paper is organized as follows. In $\S 2$, we formulate the problem. In $\S 3$, we briefly discuss the discontinuity in the velocity distribution function and give some remarks on the numerical method used in the present computation. In $\S 4$, we present numerical results for the thermal edge flow and the radiometric flow. Here, the purpose is to clarify the similarity between these flows and explain the mechanism of the radiometric flow based on it. In $\S 5$, we consider the decay properties of the flows as the system approaches the continuum limit (the limit in which the Knudsen number, defined as the ratio of the molecular mean free path to the size of the system, tends to zero). We show that the thermal edge flow vanishes at every point in the domain but the radiometric flow does not vanish at the edges in this limit. In $\S 6$, we investigate the structure of the normal stress on (and near) the plate. We show that there is a significant contribution from the thermal stress to the normal 


\section{Rarefied gas flow around a sharp edge}

stress near the edge and that the radiometric force (i.e. the force exerted on the plate from the hotter to the colder side) is caused by this effect. Section 7 is a conclusion.

\section{The problem and basic equations}

\subsection{The problem}

Let us consider a rarefied gas confined in a square vessel $-L / 2 \leqslant X_{2} \leqslant L / 2$ and $-L / 2 \leqslant X_{1} \leqslant L / 2$ maintained at a uniform temperature $T_{0}$, where $X_{i}$ is the rectangular space coordinate system (figure 1). A flat plate with width $D$ and without thickness is placed in the gas at $X_{1}=0$ and $-D / 2 \leqslant X_{2} \leqslant D / 2$. Let us denote by $S^{+}$and $S^{-}$the right- and left-hand sides of the plate, respectively, i.e.

$$
S^{ \pm}=\left\{\left(X_{1}, X_{2}\right) \mid X_{1}=0_{ \pm},-D / 2 \leqslant X_{2} \leqslant D / 2\right\}
$$

(in this paper, for a given $a, a_{+}$and $a_{-}$mean the limits from above and below, respectively, i.e. $\left.a_{ \pm}=\lim _{\epsilon(>0) \rightarrow 0}(a \pm \epsilon)\right)$. $S^{+}$and $S^{-}$are kept at uniform temperatures $T_{w}^{+}$and $T_{w}^{-}$, respectively. We investigate the steady behaviour of the gas induced in the container under the following assumptions:

(i) the behaviour of the gas is described by the BGK model of the Boltzmann equation;

(ii) the gas molecules are reflected according to the corresponding part of the equilibrium distribution having zero velocity, the temperature on the boundary and the magnitude such that there is no net mass flux across the boundary (i.e. diffuse reflection boundary condition).

In the present study, we consider the following two situations:

(A) the temperatures of both sides are the same, i.e. $T_{w}^{-}=T_{w}^{+}=T_{w}$. Here, $T_{w}\left(\neq T_{0}\right)$ denotes the uniform temperature of the plate;

(B) $S^{+}$is kept at a higher temperature than the vessel, whereas $S^{-}$is at the same temperature as the vessel, i.e. $T_{w}^{+}>T_{0}$ and $T_{w}^{-}=T_{0}$.

In case (A), the thermal edge flow is induced, whereas in case (B), it is the radiometric flow.

\subsection{Basic equations}

Let us first introduce the basic notation: $\xi_{i}$ (or $\xi$ ) denotes the molecular velocity, $f\left(X_{1}, X_{2}, \xi\right)$ the velocity distribution function, $\rho\left(X_{1}, X_{2}\right)$ the density, $v_{i}\left(X_{1}, X_{2}\right)$ the flow velocity $\left(v_{3}=0\right), T\left(X_{1}, X_{2}\right)$ the temperature, $p\left(X_{1}, X_{2}\right)$ the pressure, $p_{i j}\left(X_{1}, X_{2}\right)$ the stress tensor $\left(p_{13}=p_{31}=p_{23}=p_{32}=0\right)$ of the gas and $R$ the specific gas constant (the Boltzmann constant divided by the mass of a molecule). We also denote by $\rho_{a v}$ the average density of the gas, i.e.

$$
\rho_{a v}=\frac{1}{L^{2}} \int_{-L / 2}^{L / 2} \int_{-L / 2}^{L / 2} \rho \mathrm{d} X_{1} \mathrm{~d} X_{2} .
$$

Then, we introduce the dimensionless variables $\left(x_{i}, \zeta_{i}, \hat{f}, \hat{\rho}, \hat{v}_{i}, \hat{T}, \hat{p}, \hat{p}_{i j}\right)$ corresponding to $\left(X_{i}, \xi_{i}, f, \rho, v_{i}, T, p, p_{i j}\right)$ by the following relations:

$$
\begin{gathered}
x_{i}=\frac{X_{i}}{D}, \quad \zeta_{i}=\frac{\xi_{i}}{\left(2 R T_{0}\right)^{1 / 2}}, \quad \hat{f}=\frac{f}{\rho_{a v} /\left(2 R T_{0}\right)^{3 / 2}}, \\
\hat{\rho}=\frac{\rho}{\rho_{a v}}, \quad \hat{v}_{i}=\frac{v_{i}}{\left(2 R T_{0}\right)^{1 / 2}}, \quad \hat{T}=\frac{T}{T_{0}}, \quad \hat{p}=\frac{p}{R \rho_{a v} T_{0}}, \quad \hat{p}_{i j}=\frac{p_{i j}}{R \rho_{a v} T_{0}} .
\end{gathered}
$$




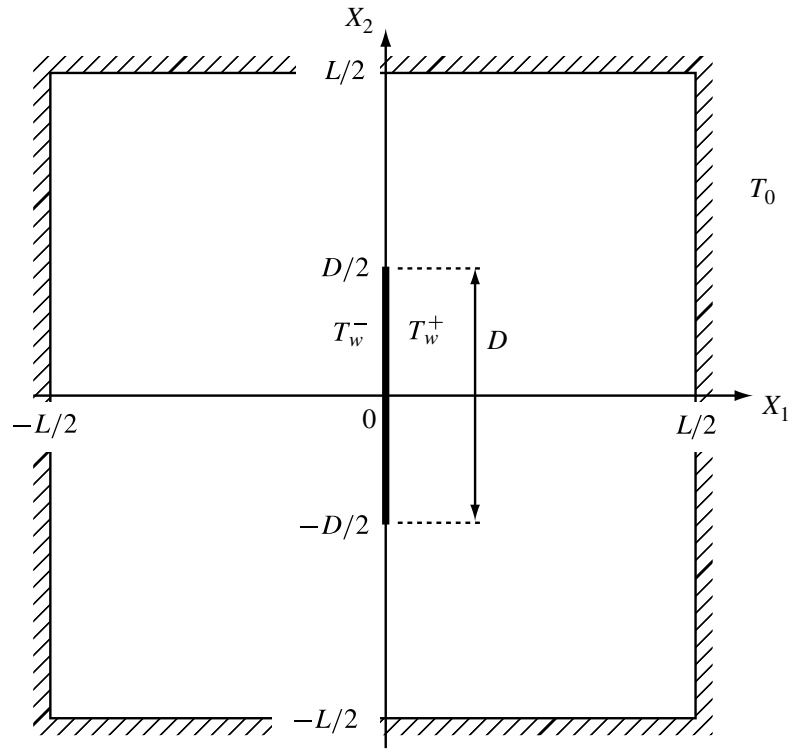

FIGURE 1. The problem.

The BGK equation for the present steady two-dimensional problem is written as

$$
\begin{gathered}
\zeta_{1} \frac{\partial \hat{f}}{\partial x_{1}}+\zeta_{2} \frac{\partial \hat{f}}{\partial x_{2}}=\frac{2}{\sqrt{\pi}} \frac{1}{K n} \hat{\rho}\left(\hat{f}_{e}-\hat{f}\right), \\
\hat{f}_{e}=\frac{\hat{\rho}}{(\pi \hat{T})^{3 / 2}} \exp \left(-\frac{\left(\zeta_{j}-\hat{v}_{j}\right)^{2}}{\hat{T}}\right), \\
\hat{\rho}=\int \hat{f} \mathrm{~d} \zeta, \quad \hat{v}_{i}=\frac{1}{\hat{\rho}} \int \zeta_{i} \hat{f} \mathrm{~d} \zeta, \quad \hat{T}=\frac{2}{3 \hat{\rho}} \int\left(\zeta_{j}-\hat{v}_{j}\right)^{2} \hat{f} \mathrm{~d} \zeta, \\
K n=\frac{\ell_{0}}{D}, \quad \ell_{0}=\frac{2}{\sqrt{\pi}} \frac{\left(2 R T_{0}\right)^{1 / 2}}{A_{c} \rho_{a v}},
\end{gathered}
$$

where $\hat{v}_{3}=0, \mathrm{~d} \zeta=\mathrm{d} \zeta_{1} \mathrm{~d} \zeta_{2} \mathrm{~d} \zeta_{3}$ and $A_{c}$ is a constant ( $A_{c} \rho$ is the collision frequency). Here and elsewhere, the domain of integration with respect to $\zeta=\left(\zeta_{1}, \zeta_{2}, \zeta_{3}\right)$ is the whole space, unless otherwise stated; $\ell_{0}$ is the mean free path of the gas molecules in the equilibrium state at rest with temperature $T_{0}$ and density $\rho_{a v}$ and $K n$ is the Knudsen number.

The diffuse reflection boundary condition on each side of the plate is given by

$$
\begin{gathered}
\hat{f}=\frac{\hat{\sigma}_{w}^{ \pm}}{\left(\pi \hat{T}_{w}^{ \pm}\right)^{3 / 2}} \exp \left(-\frac{\zeta_{j}^{2}}{\hat{T}_{w}^{ \pm}}\right) \quad \text { for } \zeta_{1} \gtrless 0 \quad\left(x_{1}=0_{ \pm},-1 / 2 \leqslant x_{2} \leqslant 1 / 2\right), \\
\hat{\sigma}_{w}^{ \pm}=\mp 2\left(\frac{\pi}{\hat{T}_{w}^{ \pm}}\right)^{1 / 2} \int_{\zeta_{1} \lessgtr 0} \zeta_{1} \hat{f}\left(x_{1}=0_{ \pm}, x_{2}, \zeta\right) \mathrm{d} \zeta, \\
\hat{T}_{w}^{ \pm}=T_{w}^{ \pm} / T_{0},
\end{gathered}
$$


where the upper (or lower) sign corresponds to the condition on $S^{+}$(or $S^{-}$). On the other hand, the boundary condition on the container is given by

$$
\begin{gathered}
\hat{f}=\frac{\hat{\sigma}_{w}}{\pi^{3 / 2}} \exp \left(-\zeta_{j}^{2}\right) \quad \text { for } \zeta_{j} n_{j}>0 \\
\left(x_{1}= \pm \hat{L} / 2,-\hat{L} / 2 \leqslant x_{2} \leqslant \hat{L} / 2\right) \text { or }\left(-\hat{L} / 2<x_{1}<\hat{L} / 2, x_{2}= \pm \hat{L} / 2\right), \\
\hat{\sigma}_{w}=-2 \sqrt{\pi} \int_{\zeta_{j} n_{j}<0} \zeta_{j} n_{j} \hat{f} \mathrm{~d} \zeta, \\
\hat{L}=L / D,
\end{gathered}
$$

where $n_{i}$ is the unit normal vector to the boundary pointing to the gas, i.e.

$$
n_{i}= \begin{cases}(\mp 1,0,0) & \left(x_{1}= \pm \hat{L} / 2,-\hat{L} / 2 \leqslant x_{2} \leqslant \hat{L} / 2\right), \\ (0, \mp 1,0) & \left(-\hat{L} / 2<x_{1}<\hat{L} / 2, x_{2}= \pm \hat{L} / 2\right) .\end{cases}
$$

Since the problem is symmetric with respect to $x_{2}=0$, we can analyse the present boundary-value problem in the upper half-domain $x_{2} \geqslant 0$ by imposing the specular reflection boundary condition at $x_{2}=0$,

$$
\hat{f}\left(x_{1}, 0, \zeta_{1}, \zeta_{2}, \zeta_{3}\right)=\hat{f}\left(x_{1}, 0, \zeta_{1},-\zeta_{2}, \zeta_{3}\right) \text { for } \zeta_{2}>0\left(-\hat{L} / 2<x_{1}<\hat{L} / 2\right) .
$$

The solution in the lower half-domain $x_{2}<0$ is then given in terms of that in the upper half-domain by

$$
\begin{gathered}
\hat{f}\left(x_{1}, x_{2}, \zeta_{1}, \zeta_{2}, \zeta_{3}\right)=\hat{f}\left(x_{1},-x_{2}, \zeta_{1},-\zeta_{2}, \zeta_{3}\right) \\
\left(-\hat{L} / 2 \leqslant x_{1} \leqslant \hat{L} / 2,-\hat{L} / 2 \leqslant x_{2}<0\right) .
\end{gathered}
$$

Finally, the pressure and the stress tensor are given by

$$
\hat{p}=\hat{\rho} \hat{T}, \quad \hat{p}_{i j}=2 \int\left(\zeta_{i}-\hat{v}_{i}\right)\left(\zeta_{j}-\hat{v}_{j}\right) \hat{f} \mathrm{~d} \zeta .
$$

To summarize, the problem is characterized by the following dimensionless parameters:

$$
K n=\frac{\ell_{0}}{D}, \quad \hat{T}_{w}^{+}=\frac{T_{w}^{+}}{T_{0}}, \quad \hat{T}_{w}^{-}=\frac{T_{w}^{-}}{T_{0}}, \quad \hat{L}=\frac{L}{D},
$$

where $\hat{T}_{w}^{+}=\hat{T}_{w}^{-}(\neq 1)$ in the case of the thermal edge flow (case (A) in $\left.\S 2.1\right)$ and $\hat{T}_{w}^{+}>1$ and $\hat{T}_{w}^{-}=1$ in the case of the radiometric flow (case (B) in $\S 2.1$ ).

\subsection{Further transformations}

Following the standard procedure (Chu 1965; Sone 2007), we can eliminate the $X_{3}$ component of the molecular velocity $\zeta_{3}$ from the system of equations. Let us introduce the marginal velocity distribution functions by

$$
\Phi=\left[\begin{array}{l}
g \\
h
\end{array}\right]=\int_{-\infty}^{\infty}\left[\begin{array}{c}
1 \\
\zeta_{3}^{2}
\end{array}\right] \hat{f} \mathrm{~d} \zeta_{3} .
$$

Multiplying (2.5), (2.9), (2.12) and (2.16) by 1 and $\zeta_{3}^{2}$ and integrating the results with respect to $\zeta_{3}$ from $-\infty$ to $\infty$, we obtain a system of simultaneous equations 
for $g$ and $h$. The equations and boundary conditions thus obtained are summarized as follows:

$$
\begin{aligned}
& \zeta_{1} \frac{\partial \Phi}{\partial x_{1}}+\zeta_{2} \frac{\partial \Phi}{\partial x_{2}}=\frac{2}{\sqrt{\pi}} \frac{1}{K n} \hat{\rho}\left(\Phi_{e}-\Phi\right), \\
& \Phi_{e}=\left[\begin{array}{c}
2 / \hat{T} \\
1
\end{array}\right] \frac{\hat{\rho}}{2 \pi} \exp \left(-\frac{\left(\zeta_{1}-\hat{v}_{1}\right)^{2}+\left(\zeta_{2}-\hat{v}_{2}\right)^{2}}{\hat{T}}\right), \\
& \hat{\rho}=\int_{-\infty}^{\infty} \int_{-\infty}^{\infty} g \mathrm{~d} \zeta_{1} \mathrm{~d} \zeta_{2}, \quad \hat{v}_{i}=\frac{1}{\hat{\rho}} \int_{-\infty}^{\infty} \int_{-\infty}^{\infty} \zeta_{i} g \mathrm{~d} \zeta_{1} \mathrm{~d} \zeta_{2}, \\
& \hat{T}=\frac{2}{3 \hat{\rho}} \int_{-\infty}^{\infty} \int_{-\infty}^{\infty}\left\{\left[\left(\zeta_{1}-\hat{v}_{1}\right)^{2}+\left(\zeta_{2}-\hat{v}_{2}\right)^{2}\right] g+h\right\} \mathrm{d} \zeta_{1} \mathrm{~d} \zeta_{2}, \\
& \Phi=\left[\begin{array}{c}
2 / \hat{T}_{w}^{ \pm} \\
1
\end{array}\right] \frac{\hat{\sigma}_{w}^{ \pm}}{2 \pi} \exp \left(-\frac{\zeta_{1}^{2}+\zeta_{2}^{2}}{\hat{T}_{w}^{ \pm}}\right) \quad \text { for } \zeta_{1} \gtrless 0\left(x_{1}=0_{ \pm}, 0 \leqslant x_{2} \leqslant 1 / 2\right), \\
& \hat{\sigma}_{w}^{ \pm}=\mp 2\left(\frac{\pi}{\hat{T}_{w}^{ \pm}}\right)^{1 / 2} \int_{\zeta_{1} \lessgtr 0} \zeta_{1} g\left(x_{1}=0_{ \pm}, x_{2}, \zeta_{1}, \zeta_{2}\right) \mathrm{d} \zeta_{1} \mathrm{~d} \zeta_{2}, \\
& \Phi=\left[\begin{array}{l}
2 \\
1
\end{array}\right] \frac{\hat{\sigma}_{w}}{2 \pi} \exp \left(-\left(\zeta_{1}^{2}+\zeta_{2}^{2}\right)\right) \text { for } \zeta_{1} n_{1}+\zeta_{2} n_{2}>0 \\
& \left(x_{1}= \pm \hat{L} / 2,0 \leqslant x_{2} \leqslant \hat{L} / 2\right) \text { or }\left(-\hat{L} / 2<x_{1}<\hat{L} / 2, x_{2}=\hat{L} / 2\right) \text {, } \\
& \hat{\sigma}_{w}=-2 \sqrt{\pi} \int_{\zeta_{1} n_{1}+\zeta_{2} n_{2}<0}\left(\zeta_{1} n_{1}+\zeta_{2} n_{2}\right) g \mathrm{~d} \zeta_{1} \mathrm{~d} \zeta_{2}, \\
& \Phi\left(x_{1}, 0, \zeta_{1}, \zeta_{2}\right)=\Phi\left(x_{1}, 0, \zeta_{1},-\zeta_{2}\right) \text { for } \zeta_{2}>0\left(-\hat{L} / 2<x_{1}<\hat{L} / 2\right) .
\end{aligned}
$$

Here, the range of $x_{2}$ has been restricted to $0 \leqslant x_{2} \leqslant \hat{L} / 2$ by the use of the specular boundary condition (2.29). The stress tensor is expressed in terms of $g$ and $h$ as

$$
\begin{gathered}
\hat{p}_{i j}=2 \int_{-\infty}^{\infty} \int_{-\infty}^{\infty}\left(\zeta_{i}-\hat{v}_{i}\right)\left(\zeta_{j}-\hat{v}_{j}\right) g \mathrm{~d} \zeta_{1} \mathrm{~d} \zeta_{2} \quad(i, j=1,2), \\
\hat{p}_{33}=2 \int_{-\infty}^{\infty} \int_{-\infty}^{\infty} h \mathrm{~d} \zeta_{1} \mathrm{~d} \zeta_{2} .
\end{gathered}
$$

\section{Numerical analysis}

An important feature of the present problem is that the presence of the edges introduces discontinuities in the velocity distribution function. This is closely related to the abrupt variation of the macroscopic quantities near the edges whose correct description is essential for the purpose of the present study. Below, we first discuss the discontinuity and then give some remarks on the numerical method.

\subsection{Discontinuity in the velocity distribution function}

As discussed in Sone \& Takata (1992), if the velocity distribution function is discontinuous at a certain molecular velocity at a certain location in the space, the discontinuity propagates from this location along the characteristic of (2.21). Such a propagation of the discontinuity is commonly observed in a gas around a convex boundary, and the velocity distribution function is generally discontinuous there. In the 
present problem, the convex nature of the boundary is concentrated at the edges of the plate, and discontinuities are introduced there, as explained below.

Let us consider the velocity distribution of the gas molecules coming into the positive half-domain $\left(x_{1}>0\right)$ or the negative half-domain $\left(x_{1}<0\right)$ by either leaving the plate surface $\left(x_{2}<1 / 2\right)$ or by passing through the $x_{2}$ axis $\left(x_{2}>1 / 2\right)$. At the location of the edge, $\left(x_{1}, x_{2}\right)=(0,1 / 2)$, the limit of $\Phi$ from below, $\Phi\left(x_{1}=0_{ \pm}, x_{2}=\right.$ $\left.1 / 2_{-}, \zeta_{1}, \zeta_{2}\right)$ for $\zeta_{1} \gtrless 0$, is prescribed by the diffuse reflection condition (2.25) with (2.26), whereas the limit from above, $\Phi\left(x_{1}=0, x_{2}=1 / 2_{+}, \zeta_{1}, \zeta_{2}\right)$, is determined by the state of the gas (as a solution of the kinetic equation). The nature of these velocity distributions is quite different and the velocity distribution function becomes discontinuous at the edge $\left(x_{1}, x_{2}\right)=(0,1 / 2)$ for any fixed molecular velocity $\left(\zeta_{1}, \zeta_{2}\right)$. This discontinuity propagates in the gas in the direction of $\left(\zeta_{1}, \zeta_{2}\right)$ (or along the characteristic of (2.21)). Therefore, at a point $\left(x_{1}, x_{2}\right)$ in the gas, the velocity distribution function is discontinuous in the direction $\zeta_{2} / \zeta_{1}=\left(x_{2}-1 / 2\right) / x_{1}$ in the $\left(\zeta_{1}, \zeta_{2}\right)$ plane. It should be noted that, in the framework of the boundary-value problem (2.21)-(2.29), there is another discontinuity propagating from $\left(x_{1}, x_{2}\right)=(0,1 / 2)$ in the direction $\zeta_{2} / \zeta_{1}=-\left(x_{2}+1 / 2\right) / x_{1}$, reflected at the specularly reflecting boundary $x_{2}=0$, and reaching the point $\left(x_{1}, x_{2}\right)$. Thus, the velocity distribution function at $\left(x_{1}, x_{2}\right)$ is also discontinuous in the direction $\zeta_{2} / \zeta_{1}=\left(x_{2}+1 / 2\right) / x_{1}$ in the $\left(\zeta_{1}, \zeta_{2}\right)$ plane. This second discontinuity, of course, corresponds to the one originating from the other edge $\left(x_{1}, x_{2}\right)=(0,-1 / 2)$ in the original problem not restricted to positive $x_{2}$. Owing to the effect of the molecular collisions, the discontinuity decays with the distance from the edge on the scale of the mean free path.

It may also be noted that the limits of $\hat{\sigma}_{w}$ along the wall do not generally coincide at the corner (the domain of integration in (2.28) changes discontinuously there) and this introduces another discontinuity there. However, this discontinuity is much smaller than those associated with the edges (Aoki et al. 2001b).

In Sone \& Takata (1992) the propagation of discontinuities in the velocity distribution function is discussed theoretically in connection with the S-layer (Sone 1973) appearing at the bottom of the Knudsen layer on the convex body when the Knudsen number is small. The paper by Cercignani (2000) should also be mentioned as a related work. A mathematical study of the propagation of boundaryinduced discontinuities in the solution of a simple kinetic equation is found in Aoki et al. (2001a). Also, quite recently a mathematical description of the formation and propagation of a discontinuity (in a gas in a non-convex domain) was given for the solution of the Boltzmann equation by Kim (2011).

\subsection{Remarks on the numerical analysis}

We solve the boundary-value problem (2.21)-(2.29) by the finite-difference method. The manner of propagation of the discontinuity from the edge is essentially the same as that of the leading-edge problem of a rarefied gas, for which a finite-difference method capable of describing the discontinuity accurately was devised by Aoki, Kanba \& Takata (1997). Therefore, we can employ this method with suitable adjustment. The procedure is outlined in appendix A. Essentially, it consists of dividing the domain $-\hat{L} / 2 \leqslant x_{1} \leqslant \hat{L} / 2$ and $0 \leqslant x_{2} \leqslant \hat{L} / 2$ by the lines of discontinuity and applying the finite-difference formula in each subdomain so that the finite-difference approximation is not applied across the line of discontinuity; otherwise, a large numerical error will be introduced. It should also be mentioned that the method in Aoki et al. (1997) is based on the methods developed and used in earlier works (e.g. Sone \& Sugimoto 

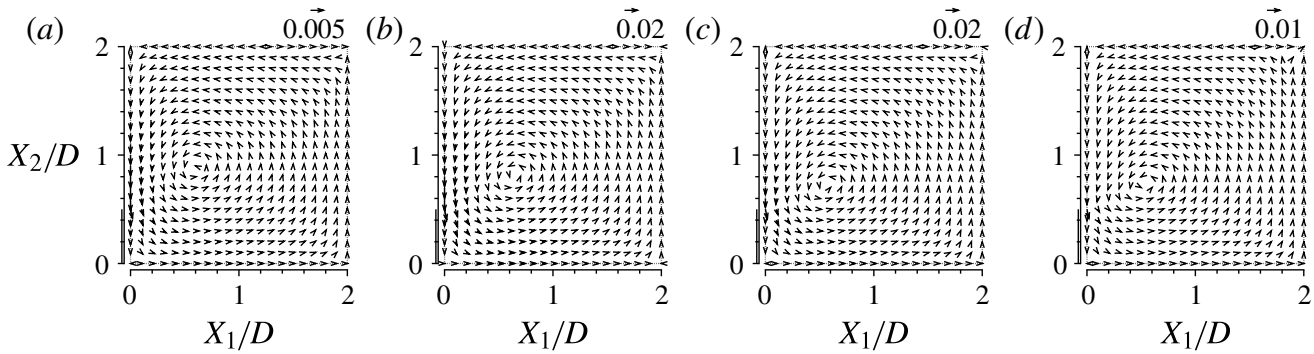

FIgURE 2. Thermal edge flow $\left(T_{w}^{+} / T_{0}=T_{w}^{-} / T_{0}=2\right.$ and $\left.L / D=4\right)$. (a) $K n=5$, (b) $K n=0.5$, (c) $K n=0.05,(d) K n=0.01$. The arrows indicate the flow velocity vector $\left(v_{1}, v_{2}\right) /\left(2 R T_{0}\right)^{1 / 2}$ at its starting point. The reference length is shown above each plot.
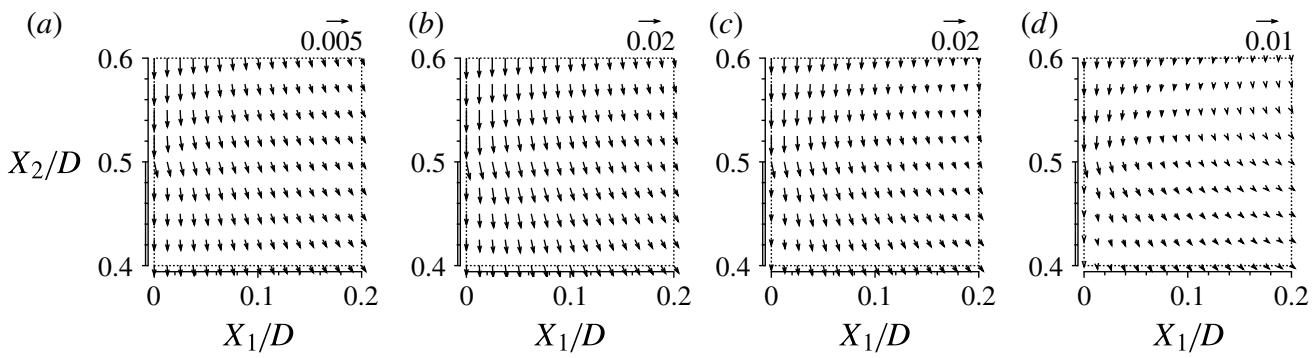

FIGURE 3. Close-up of figure 2 near the edge.

1990; Aoki et al. 1991; Sugimoto \& Sone 1992; Sone \& Sugimoto 1993; Takata, Sone \& Aoki 1993) on various problems in which the discontinuity in the velocity distribution function plays an important role.

The computations were carried out for $\hat{T}_{w}^{+}=\hat{T}_{w}^{-}=2$ and 0.5 in the case of the thermal edge flow and for $\hat{T}_{w}^{+}=2$ and $\hat{T}_{w}^{-}=1$ in the case of the radiometric flow. $\hat{L}$ has been fixed to $\hat{L}=4$ throughout the computations. $K n$ ranges from 0.01 to 20 for $\hat{T}_{w}^{+}=\hat{T}_{w}^{-}=2$ and from 0.02 to 20 for $\hat{T}_{w}^{+}=\hat{T}_{w}^{-}=0.5$ in the case of the thermal edge flow and from 0.005 to 20 in the case of the radiometric flow $\left(\hat{T}_{w}^{+}=2\right.$ and $\left.\hat{T}_{w}^{-}=1\right)$. Some data on the accuracy of the present computations are given in appendix $\mathrm{B}$.

\section{Similarity between the radiometric flow and the thermal edge flow}

We now show the numerical results obtained by the finite-difference method described above. We first show some typical flow patterns of the thermal edge and radiometric flows, and then compare the flow structures.

\subsection{Thermal edge flow}

Figures 2-7 show the flow fields of the thermal edge flow induced around a heated plate in the case of $T_{w}^{+} / T_{0}=T_{w}^{-} / T_{0}=2$ for $K n=5,0.5,0.05$ and $0.01(L / D=4)$. More precisely, figure 2 shows the flow velocity vector $\left(v_{1}, v_{2}\right)$, figure 3 its close-up near the edge and figures 4-7 the distributions of the flow speed, density, temperature and pressure, respectively. Note that the flow field is symmetric not only with respect to the $X_{1}$ axis, but also with respect to the $X_{2}$ axis. For this reason, the result is shown in the first quadrant of the domain. It should also be noted that the direction along which the discontinuity in the velocity distribution function propagates is not 
Rarefied gas flow around a sharp edge
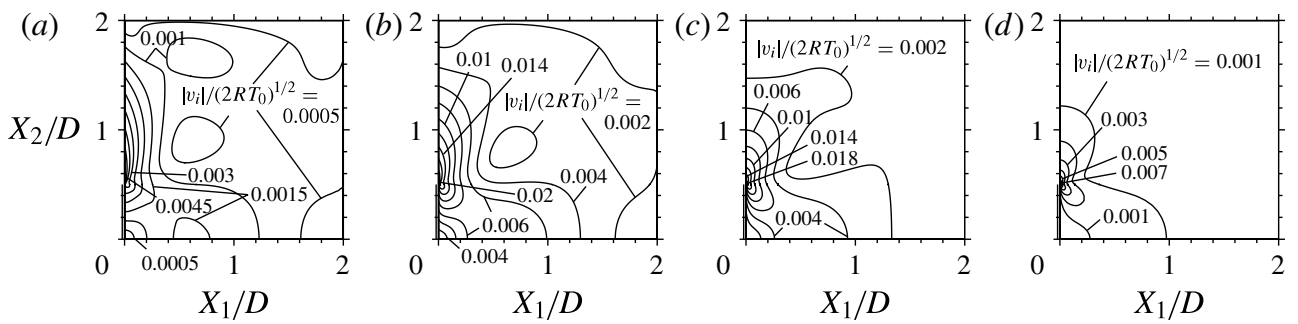

FIGURE 4. Isolines of the flow speed $\left|v_{i}\right|=\left(v_{1}^{2}+v_{2}^{2}\right)^{1 / 2}$ for the thermal edge flow $\left(T_{w}^{+} / T_{0}=T_{w}^{-} / T_{0}=2\right.$ and $\left.L / D=4\right) .(a) K n=5\left(\left|v_{i}\right| /\left(2 R T_{0}\right)^{1 / 2}=0.0005 m ; m=1,2, \ldots, 9\right)$, (b) $K n=0.5\left(\left|v_{i}\right| /\left(2 R T_{0}\right)^{1 / 2}=0.002 m ; m=1,2, \ldots, 10\right),(c) K n=0.05\left(\left|v_{i}\right| /\left(2 R T_{0}\right)^{1 / 2}=\right.$ $0.002 m ; m=1,2, \ldots, 9),(d) K n=0.01\left(\left|v_{i}\right| /\left(2 R T_{0}\right)^{1 / 2}=0.001 m ; m=1,2, \ldots, 8\right)$.
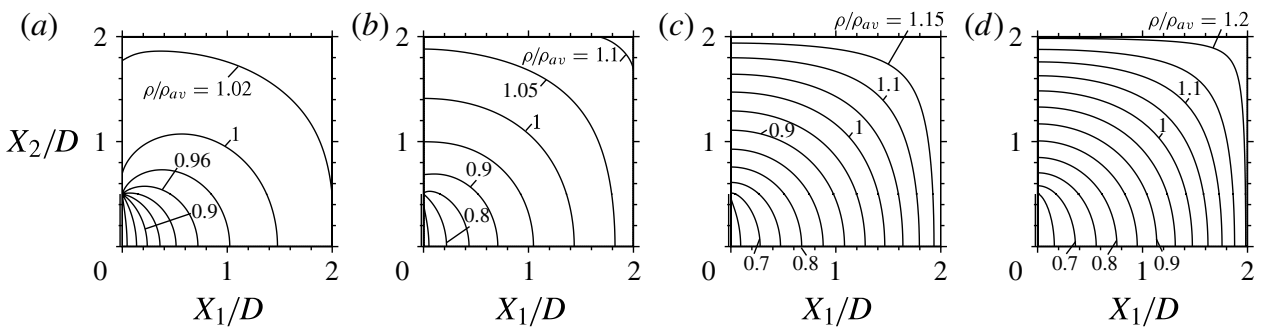

FIGURE 5. Isolines of the density for the thermal edge flow $\left(T_{w}^{+} / T_{0}=T_{w}^{-} / T_{0}=2\right.$ and $L / D=$ 4). (a) $K n=5\left(\rho / \rho_{a v}=0.86+0.02 m ; m=0,1, \ldots, 8\right),(b) K n=0.5\left(\rho / \rho_{a v}=0.75+0.05 m\right.$; $m=0,1, \ldots, 7),(c) K n=0.05\left(\rho / \rho_{a v}=0.65+0.05 m ; m=0,1, \ldots, 10\right),(d) K n=0.01$ $\left(\rho / \rho_{a v}=0.65+0.05 m ; m=0,1, \ldots, 11\right)$.
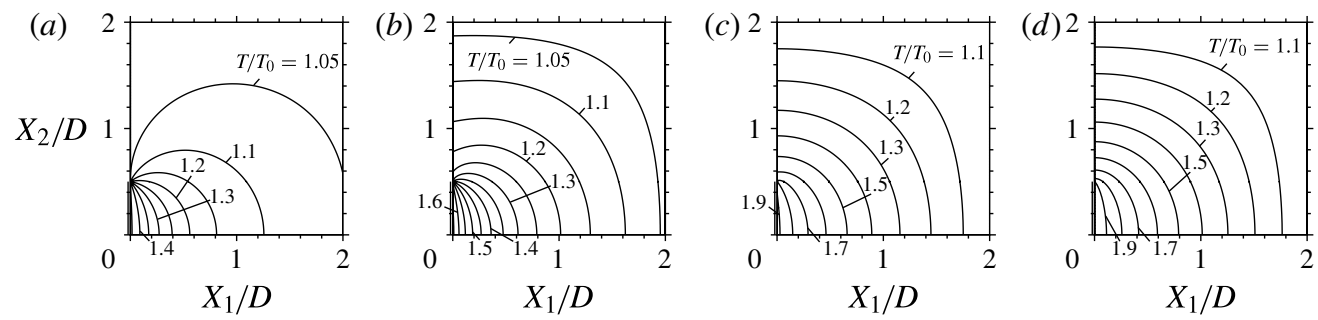

FIGURE 6. Isolines of the temperature for the thermal edge flow $\left(T_{w}^{+} / T_{0}=T_{w}^{-} / T_{0}=2\right.$ and $L / D=4)$. (a) $K n=5\left(T / T_{0}=1.05+0.05 m ; m=0,1, \ldots, 8\right),(b) K n=0.5^{w}\left(T / T_{0}=\right.$ $1.05+0.05 m ; m=0,1, \ldots, 12),(c) K n=0.05\left(T / T_{0}=1.1+0.1 m ; m=0,1, \ldots, 8\right)$, (d) $K n=0.01\left(T / T_{0}=1.1+0.1 m ; m=0,1, \ldots, 8\right)$.

determined on the edge, unless the direction of approach to it is specified. This means that the limiting velocity distribution function is different depending on the direction of approach to the edge. As a result, the macroscopic quantities are not uniquely determined at the edge. The flow velocities shown at the edge in figures 2 and 3 are those of the limiting values from the right along the line $X_{2} / D=0.5$.

For each value of $K n$, a counterclockwise circulating flow is induced in the domain. For $K n=5$, the flow is very weak. Indeed, it vanishes in the free molecular limit $K n \rightarrow \infty$, as proved by Sone $(1984,1985)$ (the proof is more general; it allows the 

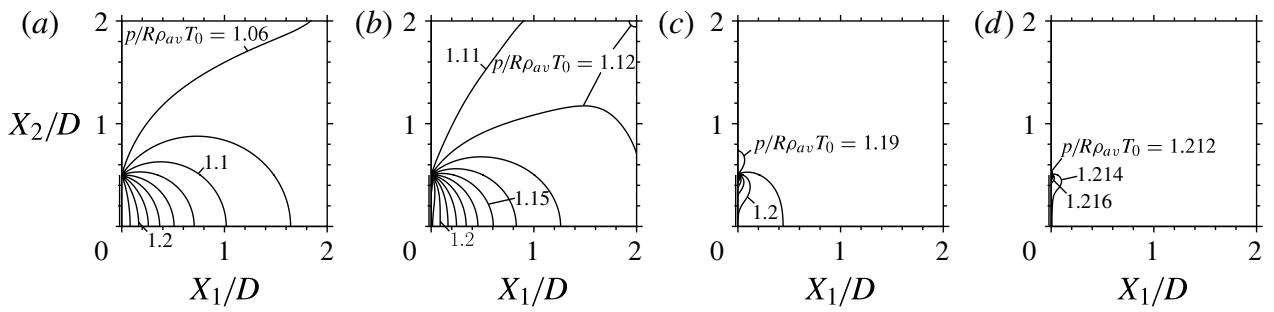

FIGURE 7. Isolines of the pressure for the thermal edge flow $\left(T_{w}^{+} / T_{0}=T_{w}^{-} / T_{0}=2\right.$ and $L / D=4)$. (a) $K n=5\left(p / R \rho_{a v} T_{0}=1.06+0.01 m ; m=0, \ldots, 8\right),(b) K n=0.5\left(p / R \rho_{a v} T_{0}=\right.$ $1.11+0.005 m ; m=0, \ldots, 12),(c) K n=0.05\left(p / R \rho_{a v} T_{0}=1.19+0.005 m ; m=0, \ldots, 8\right)$, (d) $K n=0.01\left(p / R \rho_{a v} T_{0}=1.212+0.002 m ; m=0,1, \ldots, 9\right)$.
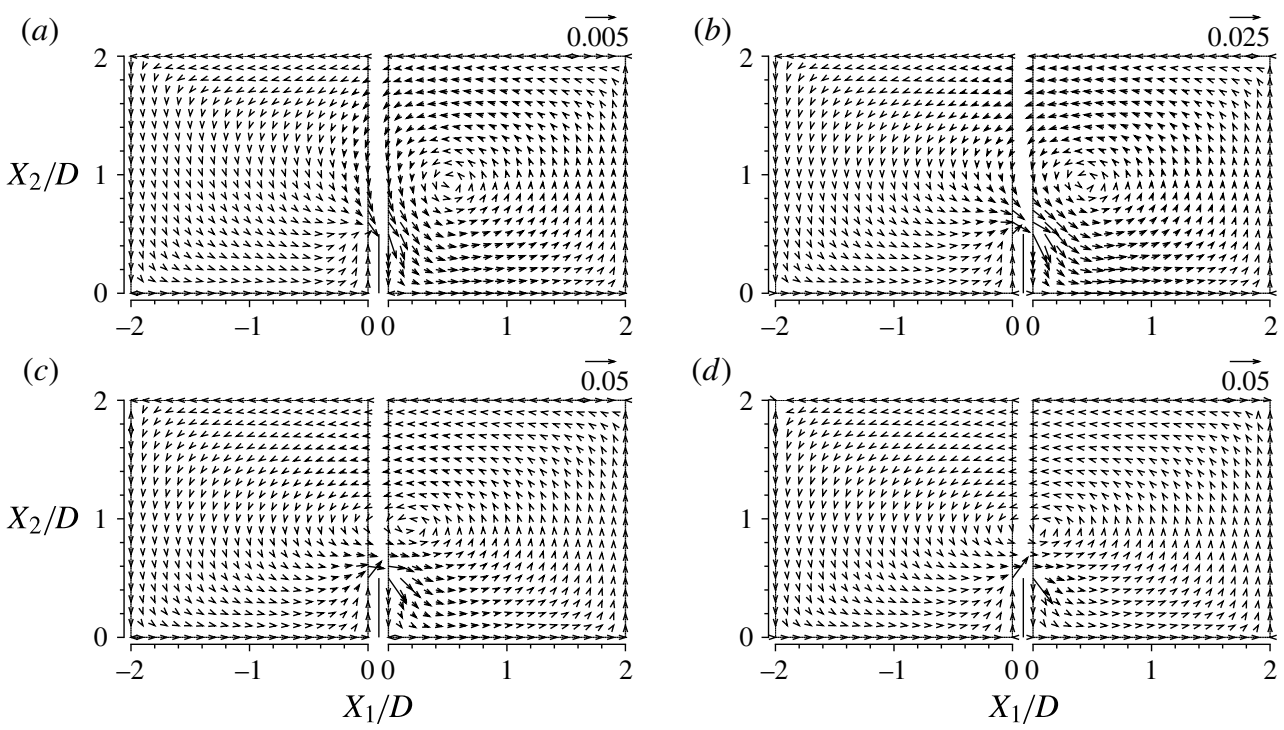

FIgURE 8. Radiometric flow $\left(T_{w}^{+} / T_{0}=2, T_{w}^{-} / T_{0}=1\right.$ and $\left.L / D=4\right)$. (a) $K n=5$, (b) $K n=0.5$, (c) $K n=0.05,(d) K n=0.01$. The flow fields for $X_{1} \gtrless 0$ are shown separately. For more details see the caption of figure 2.

shape and temperature distribution on the boundary to be arbitrary and the Maxwelltype boundary condition is assumed). As $K n$ is decreased, the flow becomes faster, attaining the highest speed around $K n=0.5$. For still smaller values of $K n$, the flow becomes weaker with the decrease of $K n$. At each $K n$, the flow speed varies substantially over the domain and it is fastest near the edge (figures 3 and 4). For the temperature, a low-temperature region appears above the plate when $K n$ is large (figure $6 a$ ). This is because the molecules present in this region are mostly the slow molecules directly coming from the wall of the container. The slow molecules are thermalised owing to the molecular collisions with the fast molecules arriving from the hot plate. As a result, the temperature above the plate increases with the decrease of Kn. These features have already been observed by Aoki et al. (1995), in which a DSMC simulation for essentially the same problem has been carried out (in the case of a cooled plate and thus the effect manifests itself in the opposite sense from the present case). The pressure exhibits a large spatial variation when 
Rarefied gas flow around a sharp edge
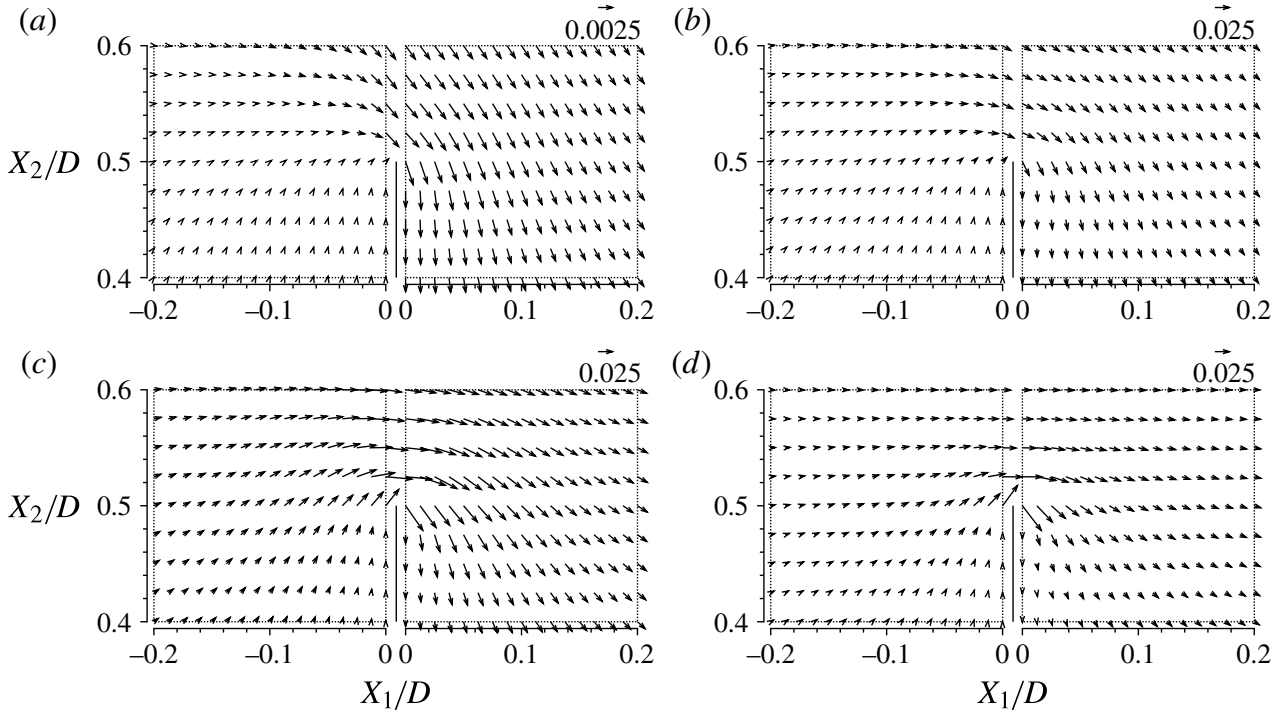

FIGURE 9. Close-up of figure 8 near the edge.

$K n$ is large (figure $7 a$ ); the pressure rises in the region next to the plate, whereas a region with lower pressure is established above the plate. Again these reflect directly the population of the fast (or thermalised) molecules present in each region. As $K n$ becomes small, the pressure approaches a uniform distribution. The speed of approach, however, is not uniform over the domain and is particularly slow near the edge.

The thermal edge flow has various potential engineering applications like other thermally driven flows. A prototype of a thermal pump driven by the thermal edge flow (the thermal edge pump) has been assembled and tested in Sugimoto \& Sone (2005). It was also demonstrated that the pump has a gas separation effect when applied to a gas mixture (Sugimoto 2009). On the other hand, a blower driven by the thermal edge flow (the thermal edge blower) has been devised and shown to be applicable to freeze drying (Kitamura et al. 2009).

\subsection{Radiometric flow}

Figures 8-13 are the corresponding figures for the radiometric flow in the case of $T_{w}^{+} / T_{0}=2$ and $T_{w}^{-} / T_{0}=1$, showing its typical flow patterns $(K n=5,0.5,0.05$ and $0.01)$; figure 8 shows the flow velocity vector $\left(v_{1}, v_{2}\right)$, figure 9 its close-up around the edge and figures 10-13 the distributions of the flow speed, density, temperature and pressure, respectively, in the upper half of the container. In figures 8 and 9, the flow fields for the regions $X_{1}>0$ and $X_{1}<0$ are shown separately to make it possible to distinguish the arrows located on both sides of the plate. Note that, as in the case of the thermal edge flow, the macroscopic quantities are not unique at the edge and they depend on the direction of approach to it (see the first paragraph of $\S 4.1$ ). The flow velocities at the edge shown in figures 8 and 9 are those of the limiting values along the line $X_{2} / D=0.5$ from the right or the left, depending on the domain $X_{1}>0$ or $X_{1}<0$.

For each $K n$, a counterclockwise circulating flow is induced. As $K n$ is reduced, the overall flow speed increases $(K n=5 \rightarrow 0.5)$, and then decreases $(K n=0.5 \rightarrow 0.05 \rightarrow$ 0.01 ) as a whole. Near the edge, in contrast, the flow speed continues to increase with 

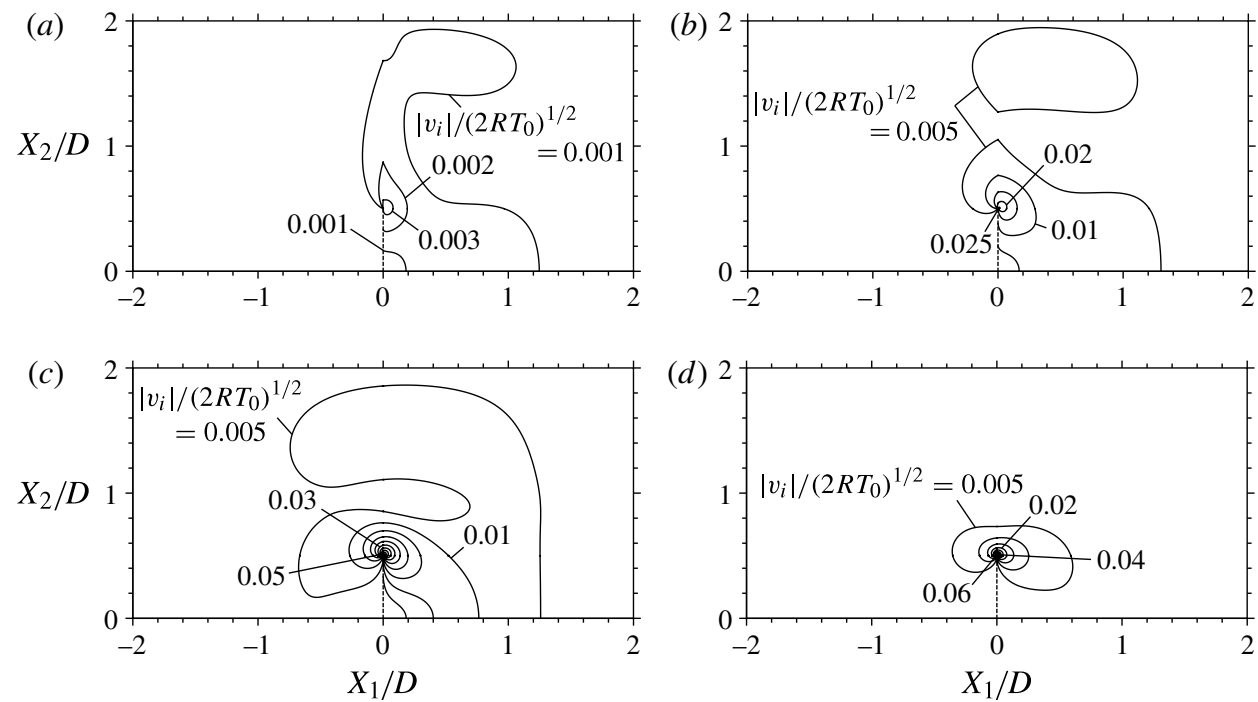

FIGURE 10. Isolines of the flow speed $\left|v_{i}\right|=\left(v_{1}^{2}+v_{2}^{2}\right)^{1 / 2}$ for the radiometric flow $\left(T_{w}^{+} / T_{0}=\right.$ 2, $T_{w}^{-} / T_{0}=1$ and $\left.L / D=4\right)$. (a) $K n=5\left(\left|v_{i}\right| /\left(2 R T_{0}\right)^{1 / 2}=0.001 m ; m=1,2, \ldots, 3\right)$, (b) $K n=$ $0.5\left(\left|v_{i}\right| /\left(2 R T_{0}\right)^{1 / 2}=0.005 m ; m=1,2, \ldots, 5\right),(c) K n=0.05\left(\left|v_{i}\right| /\left(2 R T_{0}\right)^{1 / 2}=0.005 m\right.$; $m=1,2, \ldots, 11),(d) K n=0.01\left(\left|v_{i}\right| /\left(2 R T_{0}\right)^{1 / 2}=0.005 m ; m=1,2, \ldots, 12\right)$. The dashed line indicates the plate.

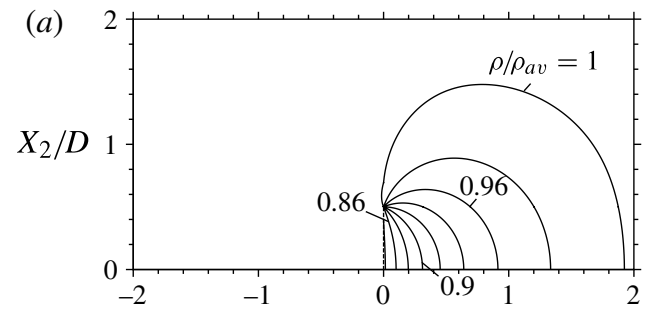

(b)
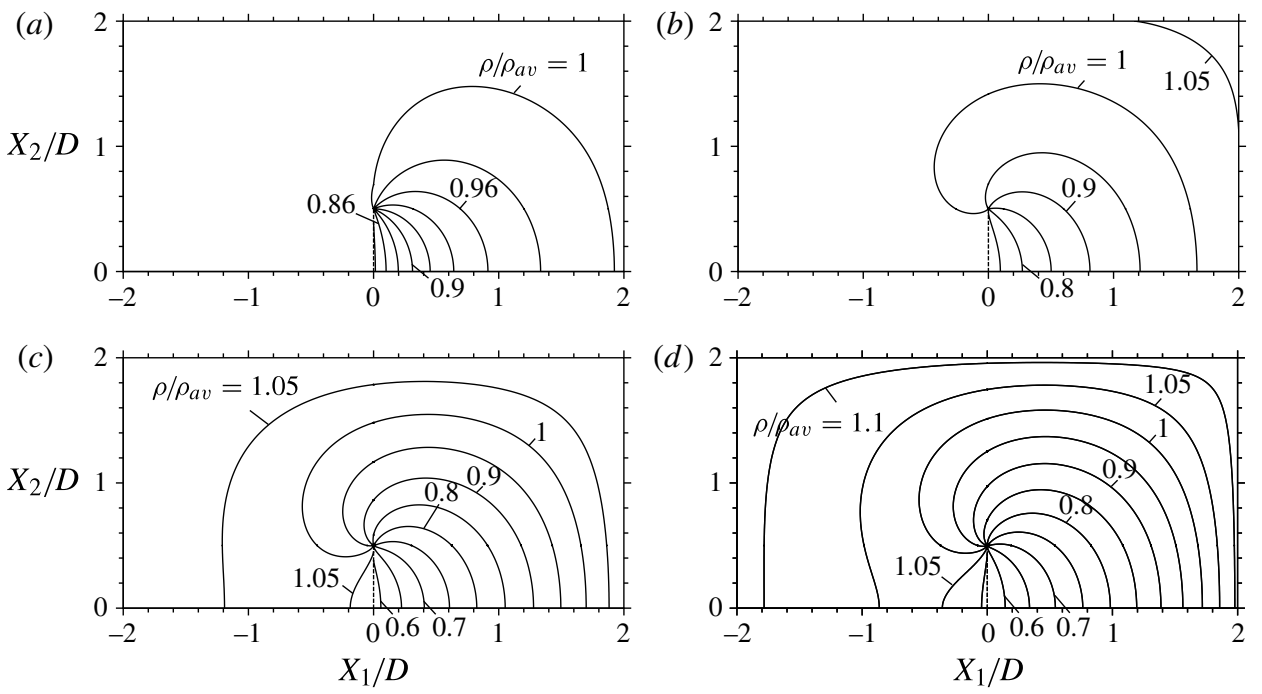

FIGURE 11. Isolines of the density for the radiometric flow $\left(T_{w}^{+} / T_{0}=2, T_{w}^{-} / T_{0}=1\right.$ and $L / D=4)$. (a) $K n=5\left(\rho / \rho_{a v}=0.84+0.02 m ; m=0,1, \ldots, 8\right),(b) K n=0.5\left(\rho / \rho_{a v}=\right.$ $0.75+0.05 m ; m=0,1, \ldots, 6),(c) K n=0.05\left(\rho / \rho_{a v}=0.6+0.05 m ; m=0,1, \ldots, 9\right)$, (d) $K n=0.01\left(\rho / \rho_{a v}=0.6+0.05 m ; m=0,1, \ldots, 10\right)$.

the decrease of $K n$ (figures 9 and 10). The variation of the flow speed throughout the domain therefore becomes larger with the decrease of $K n$. For $K n \rightarrow \infty$ (the free 
Rarefied gas flow around a sharp edge
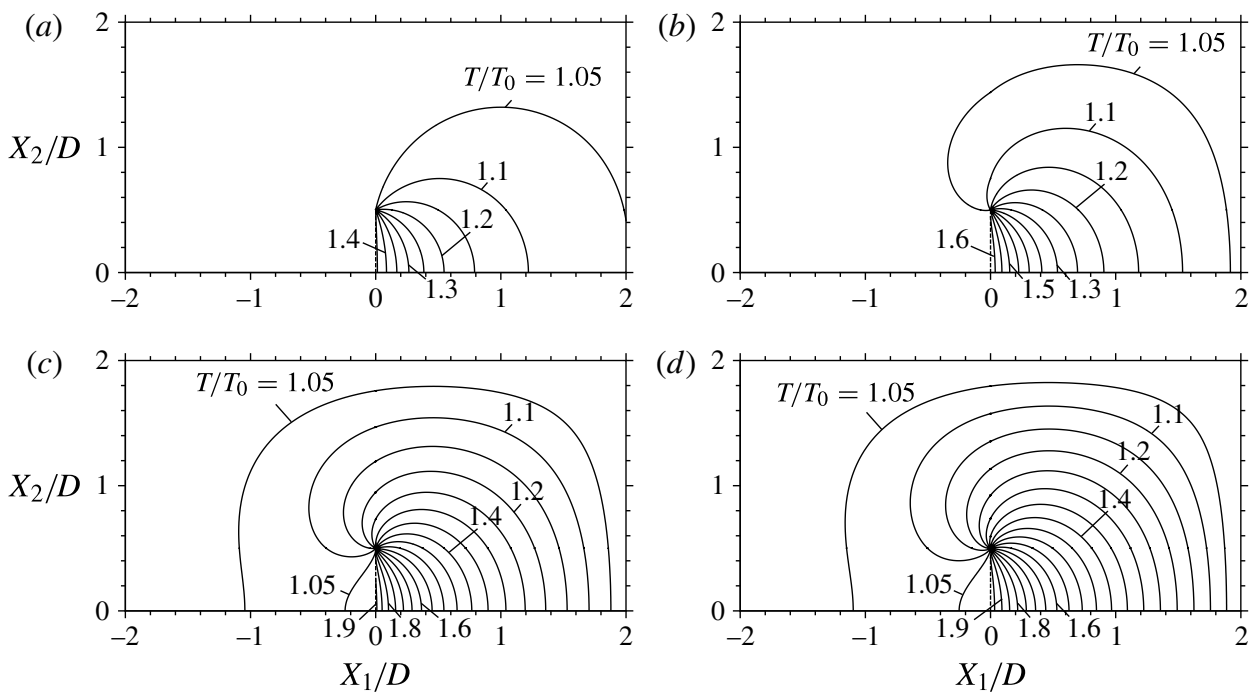

FIGURE 12. Isolines of the temperature for the radiometric flow $\left(T_{w}^{+} / T_{0}=2, T_{w}^{-} / T_{0}=1\right.$ and $L / D=4)$. (a) $K n=5\left(T / T_{0}=1.05+0.05 m ; m=0,1, \ldots, 8\right),(b) K n=0.5\left(T / T_{0}=\right.$ $1.05+0.05 m ; m=0,1, \ldots, 11),(c) K n=0.05\left(T / T_{0}=1.05+0.05 m ; m=0,1, \ldots, 17\right)$, (d) $K n=0.01\left(T / T_{0}=1.05+0.05 m ; m=0,1, \ldots, 18\right)$.
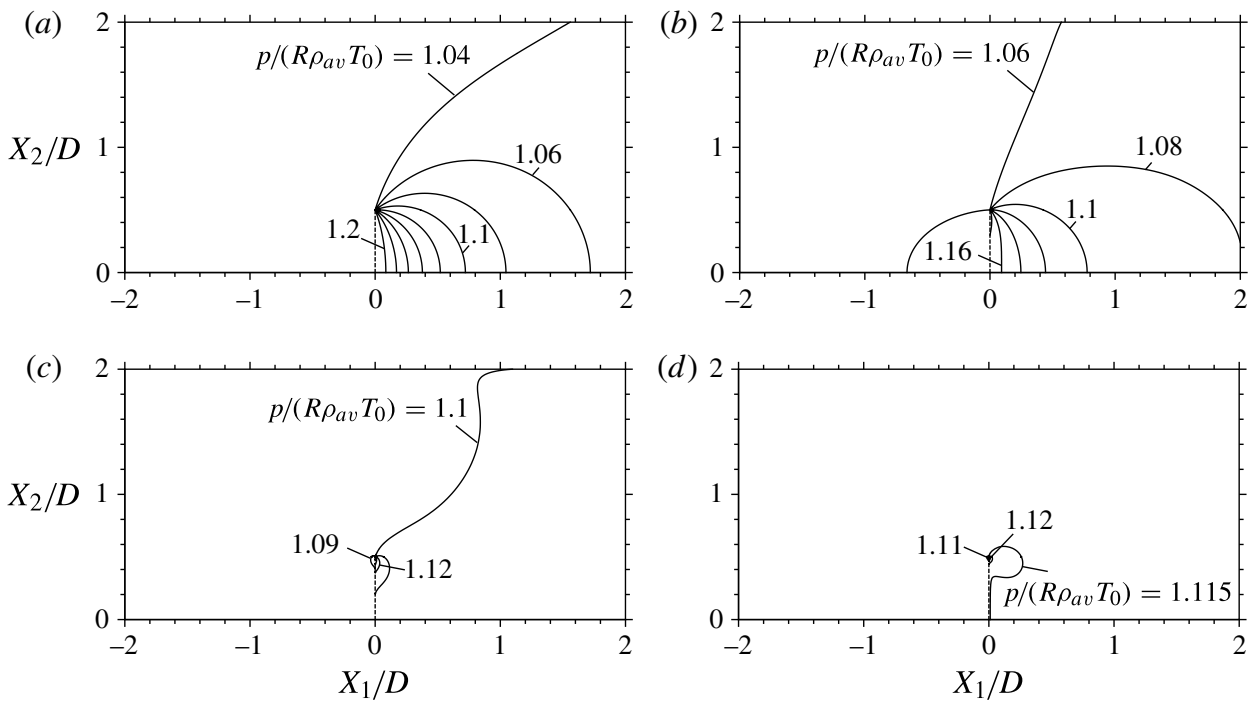

FIGURE 13. Isolines of the pressure for the radiometric flow $\left(T_{w}^{+} / T_{0}=2, T_{1} / T_{0}=2\right.$ and $L / D=4)$. (a) $K n=5\left(p / R \rho_{a v} T_{0}=1.04+0.02 m ; m=0, \ldots, 8\right),(b) K n=0.5\left(p / R \rho_{a v} T_{0}=\right.$ $1.04+0.02 m ; m=0, \ldots, 7)$, (c) $K n=0.05\left(p / R \rho_{a v} T_{0}=1.09+0.01 m ; m=0, \ldots, 5\right)$, (d) $K n=0.01\left(p / R \rho_{a v} T_{0}=1.095+0.005 m ; m=0,1, \ldots, 10\right)$.

molecular limit), no flow is induced in the container, as in the case of the thermal edge flow.

When $K n$ is large, a region with higher temperature and pressure is formed next to the plate on its right-hand side, by the effect of the thermalised molecules leaving the 
hot side of the plate (as in the case of the thermal edge flow). On the left-hand side of the plate, on the other hand, the temperature and the pressure remain unelevated. Therefore, there is a significant difference in the pressure between the right- and left-hand sides of the plate as a whole, giving rise to a net force acting on the plate from the hotter to the colder side. This is nothing but the radiometric force observed when $K n$ is large (i.e. the Knudsen regime). This explanation, however, does not apply to small $K n$, because, in this case, the pressure distribution becomes almost uniform along the plate, except in the close vicinity of the edge (again as in the case of the thermal edge flow). The cause of the radiometric force for small $K n$ must thus be sought in another mechanism, which is able to explain the structure of the pressure (or the stress) in the vicinity of the edge. We shall leave this question open until $\S 6$, in which the structure of the normal stress (to the plate) is discussed in detail.

\subsection{Similarity}

In figures 14-16, we compare the profiles of the density $\rho$, temperature $T$ and pressure $p$ of the radiometric flow with those of the thermal edge flow along four lines $X_{1} / D=0_{+}, 0.01,0.05$ and 0.1 adjacent to the plate for various $K n(K n=5,0.05$ and $0.01)$. In each figure, the top row $(a, c, e)$ shows the profiles of the thermal edge flow induced around a heated plate $\left(T_{w}^{+} / T_{0}=T_{w}^{-} / T_{0}=2\right)$ and the bottom row $(b, d, f)$ those of the radiometric flow $\left(T_{w}^{+} / T_{0}=2\right.$ and $\left.T_{w}^{-} / T_{0}=1\right)$. As one can see, each macroscopic quantity behaves in a quite analogous way along the plate in both the thermal edge and radiometric flows. For example, in both flows, the density and the pressure rise along the plate towards the edge and the temperature falls towards it. The appearance of such a similarity is not surprising when $K n$ is large, for which the flow structure of the radiometric flow in the region $X_{1}>0$ resembles that of the thermal edge flow as a whole (cf. e.g. figures $6 a$ and $12 a$ ). In the case of small $\mathrm{Kn}$, however, the observed similarity is not so obvious from the overall flow patterns. In particular, it is characteristic of both flows that a peak begins to form in the pressure near the edge $\left(X_{2} / D \sim 0.5\right)$.

Figures 17-19 show the result of the corresponding comparison, between the radiometric flow and the thermal edge flow, along four lines $X_{1} / D=0_{-},-0.01,-0.05$ and -0.1 close to the plate located on its left-hand side for $K n=5,0.05$ and 0.02 ; the top row $(a, c, e)$ shows the profiles of the density, temperature and pressure of the thermal edge flow around a cooled plate $\left(T_{w}^{+} / T_{0}=T_{w}^{-} / T_{0}=0.5\right)$ and the bottom row $(b, d, f)$ the corresponding profiles of the radiometric flow $\left(T_{w}^{+} / T_{0}=2\right.$ and $\left.T_{w}^{-} / T_{0}=1\right)$. Note that the flow fields shown for the thermal edge flow are those induced around a cooled plate. Again, each macroscopic quantity shows a similar structure along the plate in both flows (in the part $X_{2} / D \lesssim 0.5$ ), though there is a difference in the region above the plate. That is, the density and the pressure falls towards the edge along the plate, and the temperature behaves in the opposite way. Notably, a peak in the pressure is formed near the edge in both flows as in the previous comparison on the heated side, except that the peak is now directed in the negative- $p$ direction.

To summarize, the distributions of the density, temperature and pressure of the radiometric flow are similar to those of the thermal edge flow around a heated plate on the heated side of the plate and are similar to those of the thermal edge flow around a cooled plate on the unheated side. A natural implication of this is that the thermal edge flow and the radiometric flow are induced by the same underlying mechanism and thus are of the same type of the temperature-driven flow. Motivated by this thought, we now try to apply the same reasoning behind the thermal edge flow (Sone \& Yoshimoto 1997) to explain the cause of the radiometric flow. That is, along 
Rarefied gas flow around a sharp edge
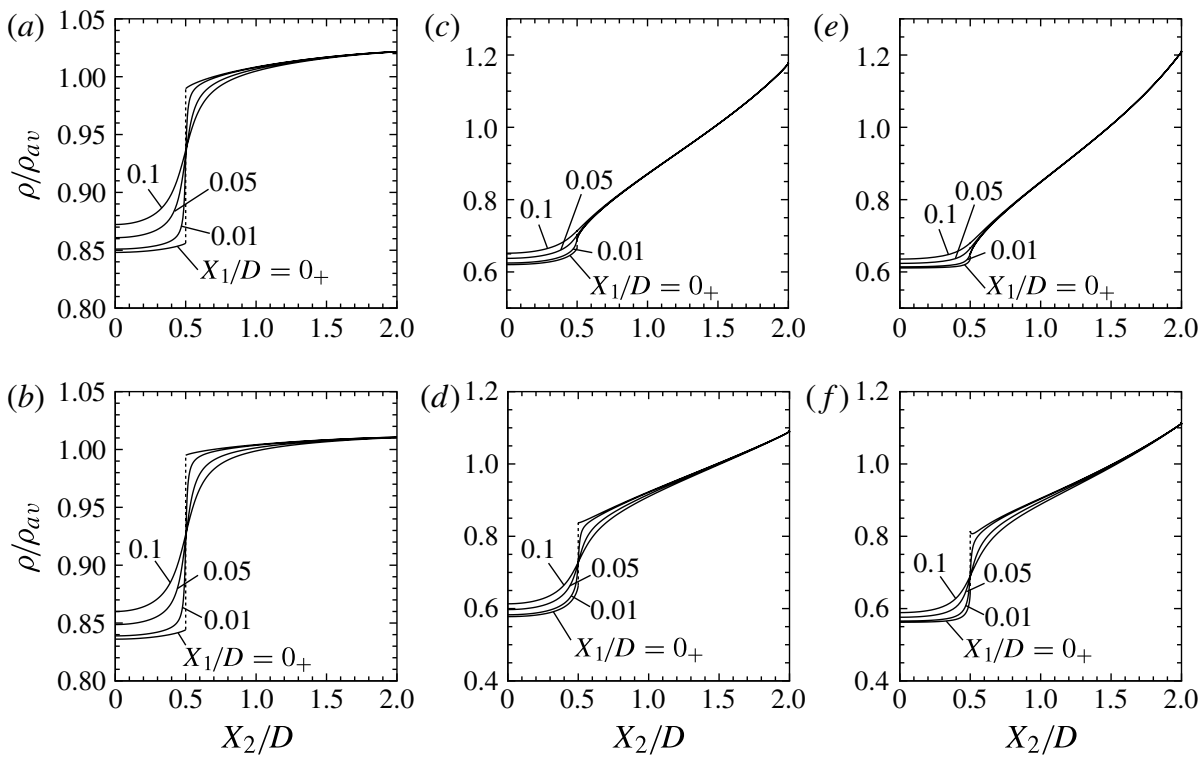

FIGURE 14. Distributions of the density $\rho$ along four lines $X_{1} / D=0_{+}, 0.01,0.05$ and 0.1 close to the plate for various $K n(L / D=4)$. Top row $(a, c, e)$ Thermal edge flow around a heated plate $\left(T_{w}^{+} / T_{0}=T_{w}^{-} / T_{0}=2\right)$; bottom row $(b, d, f)$ radiometric flow $\left(T_{w}^{+} / T_{0}=2\right.$ and $\left.T_{w}^{-} / T_{0}=1\right):(a, b) K n=5 ;(c, d) K n=0.05 ;(e, f) K n=0.01$.
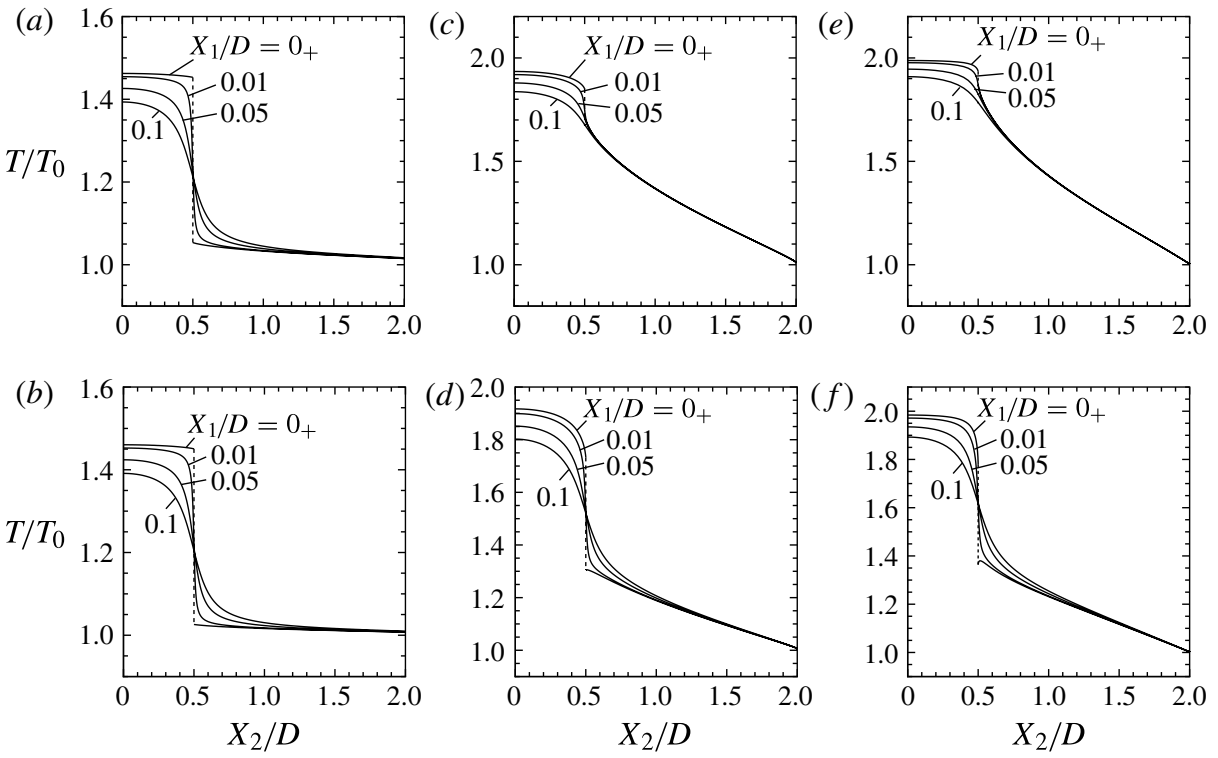

FIGURE 15. As in figure 14, but for the temperature $T:(a, b) K n=5 ;(c, d) K n=0.05$;

$(e, f) k n=0.01$.

the heated side of the plate (the right-hand side of the plate in the present problem), the temperature is decreasing towards the edge. This gives rise to a flow along (or parallel to) the plate in the downward direction (or in the direction of the temperature 

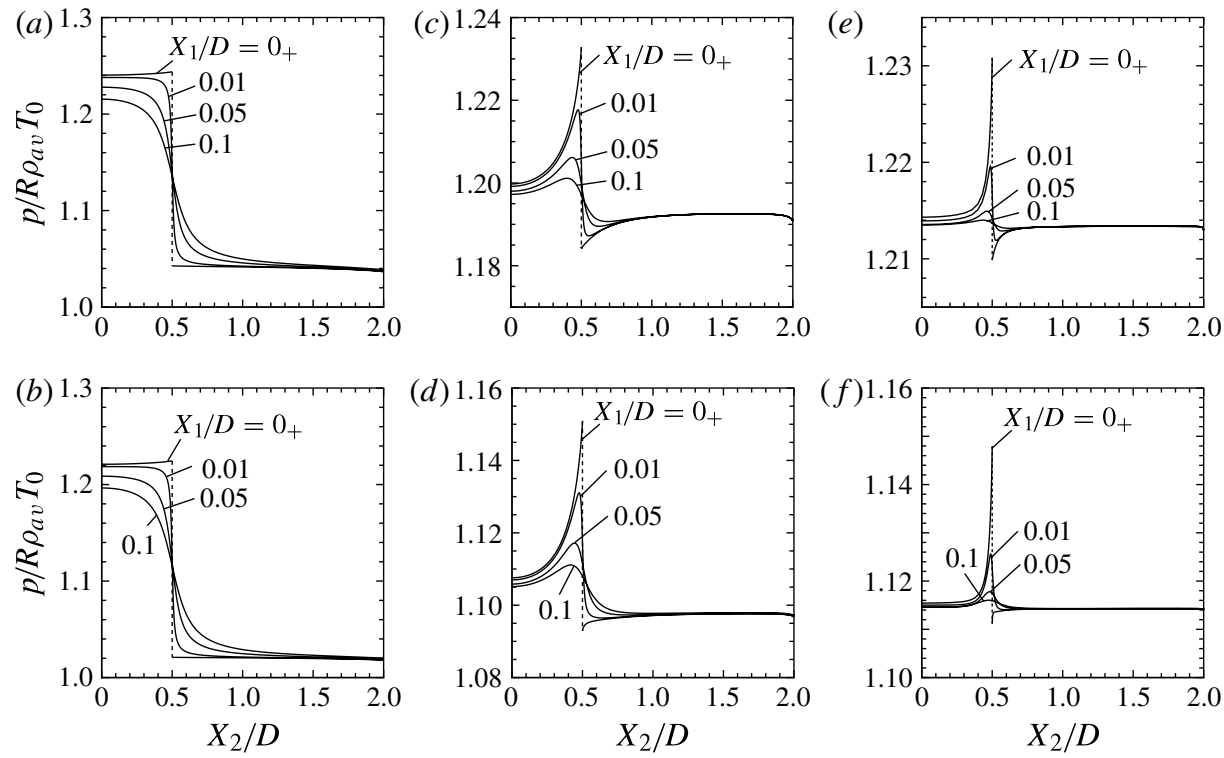

FIGURE 16. As in figure 14, but for the pressure $p:(a, b) K n=5 ;(c, d) K n=0.05$; $(e, f) K n=0.01$.
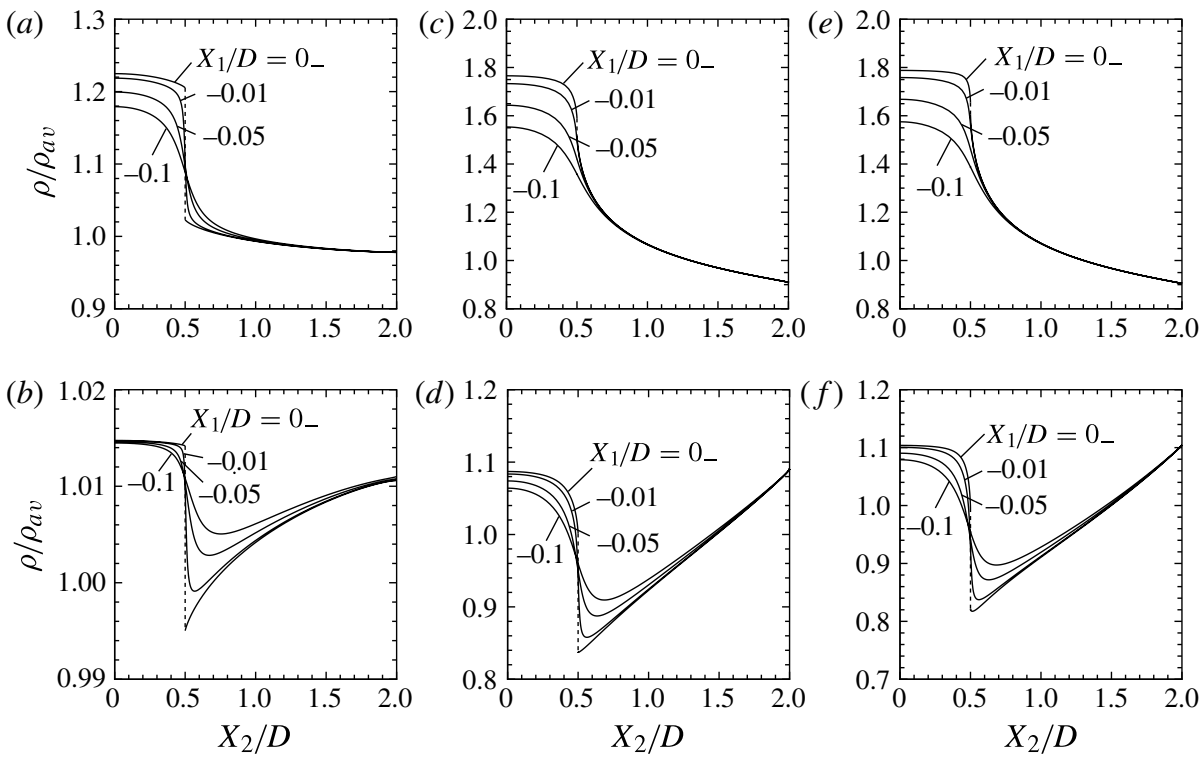

FIGURE 17. Distributions of the density $\rho$ along four lines $X_{1} / D=0_{-},-0.01,-0.05$ and -0.1 close to the plate for various $K n(L / D=4)$. Top row $(a, c, e)$ Thermal edge flow around a cooled plate $\left(T_{w}^{+} / T_{0}=T_{w}^{-} / T_{0}=0.5\right)$; bottom row $(b, d, f)$ radiometric flow $\left(T_{w}^{+} / T_{0}=2\right.$ and $\left.T_{w}^{-} / T_{0}=1\right):(a, b) K n=5 ;(c, d) K n=0.05 ;(e, f) K n=0.02$.

gradient) by the same mechanism as the thermal creep flow. On the other hand, on the unheated side of the plate (the left-hand side of the plate in the present problem), the temperature distribution is such that it is increasing towards the edge, giving rise to a 
Rarefied gas flow around a sharp edge
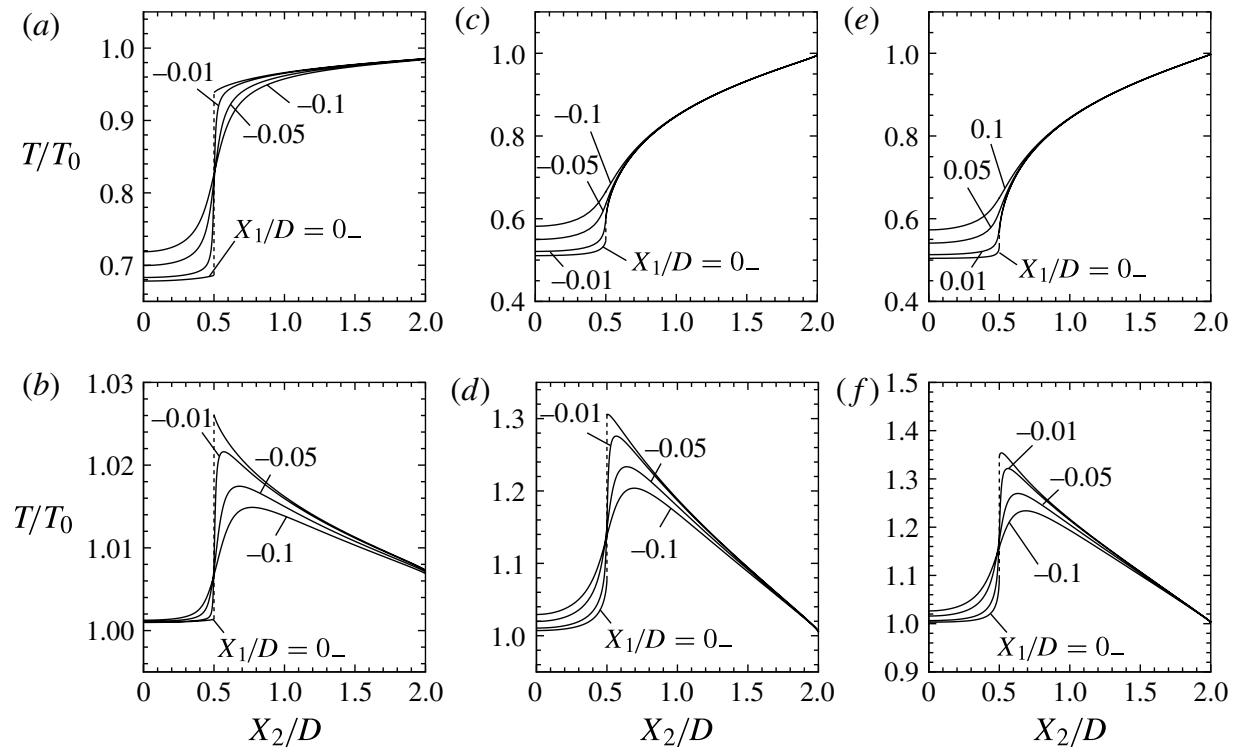

FIGURE 18. As in figure 17, but for the temperature $T:(a, b) K n=5 ;(c, d) K n=0.05$; $(e, f) K n=0.02$.
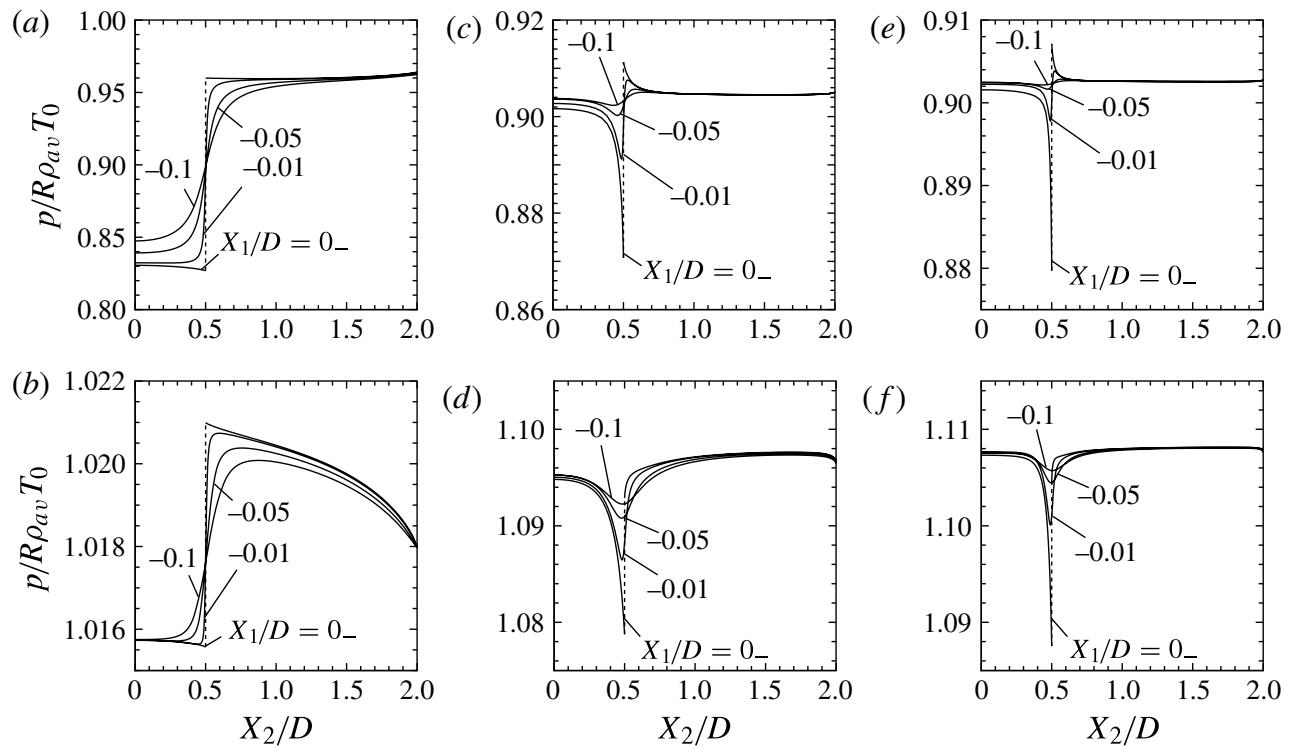

FIGURE 19. As in figure 17, but for the pressure $p:(a, b) K n=5 ;(c, d) K n=0.05$;

$$
(e, f) K n=0.02 \text {. }
$$

flow along the plate in the upward direction. Hence, there are two flows induced, one on each side, flowing in the opposite direction to each other. Such flows, once induced, cause a motion of the gas passing over the edge in the direction from the colder to the hotter side. 
Another consequence of this explanation is that it allows us to understand a feature of the flow when $K n$ is small in a natural way. In the case of the thermal edge flow, the flow becomes more localised near the edge with the decrease of $K n$. This is because there is no significant temperature variation (along the plate) in the part away from the edge (it is almost uniform along the plate; see the case $K n=0.01$ of figure $15(a, c, e)$ and the case $K n=0.02$ of figure $18(a, c, e))$. In the case of the radiometric flow, the temperature distribution along the plate also becomes flat with the decrease of $K n$, as seen from figure $15(b, d, f)$ and $18(b, d, f)$. Therefore, the flow is more and more localised near the edge with the decrease of $K n$ (cf. figure 9). In summary, the thermal edge flow and the radiometric flow are induced by the same mechanism and the temperature distribution around the edge plays an essential role.

\section{Decay property of the flows for small Knudsen numbers}

In the previous section, we have seen that the radiometric flow and the thermal edge flow belong to the same type of flow in the sense that both of them are induced by the temperature field near the edge. On the other hand, if we look at the temperature distributions shown in figures 15 and 18 carefully, we notice that the temperature variations near the edge are more abrupt in the radiometric flow than in the thermal edge flow, particularly when $K n$ is small. The temperature gradient being the driving force of the flow, there may be a difference between the two flows in the flow strength when $K n$ is small. In this section, therefore, we investigate the decay property of the flow as the Knudsen number tends to zero on the basis of the numerical result.

\subsection{Decay property of the thermal edge flow for small Knudsen numbers}

We begin with the case of the thermal edge flow. In figure 20(a,c), the variation of the flow speed $\left(v_{1}^{2}+v_{2}^{2}\right)^{1 / 2}$ with $K n$ is shown at several locations in the gas close to the edge, i.e. $\left(X_{1} / D, X_{2} / D\right)=(0.05,0.45),(0.05,0.5),(0.05,0.55)$ and $(0$, $0.55)$. Both cases in which the plate is heated $\left(T_{w}^{+} / T_{0}=T_{w}^{-} / T_{0}=2\right)$ and cooled $\left(T_{w}^{+} / T_{0}=T_{w}^{-} / T_{0}=0.5\right)$ are included in the figure. One can see that, at each location, the flow speed increases first with the decrease of $K n$, and then tends to vanish with a further decrease of $K n$. If we draw a similar plot at other point in the gas, the same behaviour is obtained. Thus, the flow vanishes in the continuum limit $K n \rightarrow 0$ at any point in the gas (except at the edge).

In order to see the limiting behaviour at the edge, we plot in figure $20(b, d)$ the variation of the same quantity at points which are away from the edge by the distance of the order of the mean free path. More precisely, the locations of the points are given by $\left(X_{1} / \ell_{0},\left(X_{2} / D-1 / 2\right) / \ell_{0}\right)=(1 / 2 \sqrt{2},-1 / 2 \sqrt{2}),(1 / 2 \sqrt{2}, 1 / 2 \sqrt{2}),(0,-1)$, $(1,0)$ and $(0,1)$; the first two points are away from the edge by the distance $\ell_{0} / 2$ and the last three points by the distance $\ell_{0}$ in the dimensional $\left(X_{1}, X_{2}\right)$ plane. It should be noted that these points are fixed in terms of the coordinates scaled by the mean free path (centred at the edge). Therefore, they approach the edge with the decrease of $K n$ in the original coordinates $\left(X_{1}, X_{2}\right)$. In this figure, the flow speed (divided by $\sqrt{2 R T_{0}}$, which is roughly the sound speed) tends to vanish in proportion to the square root of the Knudsen number as $K n$ tends to zero; the rate of decay is obviously slower than that in (a) for a fixed point. This means that the flow is more and more localised near the edge as $K n$ becomes small (this is consistent with the discussion of the last paragraph in §4.3). The region with this localised flow converges to the edge as $K n$ tends to zero and the flow vanishes there in the same limit. In this way, the state with no flow everywhere is established in the continuum limit. 

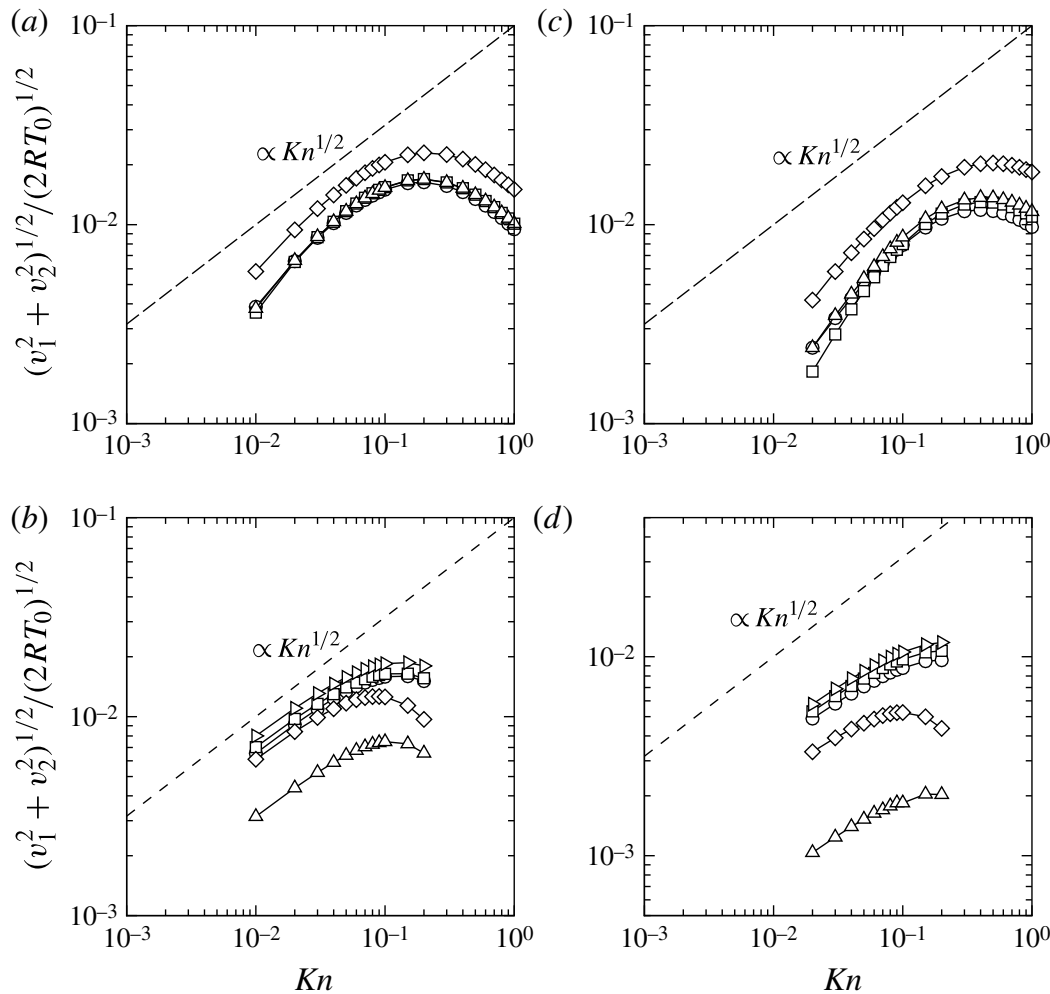

FIGURE 20. Variation of the flow speed with $K n$ of the thermal edge flow at various locations in the gas in the cases of $T_{w}^{+} / T_{0}=T_{w}^{-} / T_{0}=2$ and $0.5(L / D=4)$. (a,b) $T_{w}^{+} / T_{0}=T_{w}^{-} / T_{0}=2 ;(c, d) T_{w}^{+} / T_{0}=T_{w}^{-} / T_{0}=0.5$. In $(a, c)$, the flow speed is evaluated at four locations fixed in the gas: $\circ,\left(X_{1} / D, X_{2} / D\right)=(0.05,0.45) ; \square,(0.05,0.5) ; \triangle,(0.05$, $0.55)$; and $\diamond,(0,0.55)$. In $(b, d)$, the flow speed is evaluated at five locations in the gas having the same relative positions to the edge in the scale of the mean free path: o, $\left(X_{1} / \ell_{0},\left(X_{2} / D-1 / 2\right) / \ell_{0}\right)=(1 / 2 \sqrt{2},-1 / 2 \sqrt{2}) ; \square,(1 / 2 \sqrt{2}, 1 / 2 \sqrt{2}) ; \Delta,(0,-1) ; \diamond,(1,0) ;$ and $\triangleright,(0,1)$. The dashed line is proportional to $K n^{1 / 2}$.

It should be mentioned that Sone \& Yoshimoto (1997) gave a rough analytical estimate of the speed of the thermal edge flow. According to them, it is proportional to the square root of the mean free path. Thus, our result is consistent with theirs. The estimate is based on the classical solution of the heat-conduction equation, describing the temperature distribution of a gas over a uniformly heated semi-infinite plane. It should be noted, however, that the heat-conduction equation is not guaranteed to give a correct description of the temperature field in general even in the continuum limit (Sone et al. 1996; Sone 2002, 2007). Therefore, it requires further validation either numerically or mathematically, as mentioned in Sone \& Yoshimoto (1997). The present result provides a numerical justification for the previous estimate.

\subsection{Decay property of the radiometric flow for small Knudsen numbers}

Now we investigate the asymptotic behaviour of the radiometric flow for small $K n$. Figure 21 illustrates the variation of the local flow speed with $K n$ for this flow $\left(T_{w}^{+} / T_{0}=2\right.$ and $\left.T_{w}^{-} / T_{0}=1\right)$. As in figure 20 , we show in $(a)$ the variations at four fixed locations near the edge, given by $\left(X_{1} / D, X_{2} / D\right)=(0.05,0.45),(0.05,0.55)$, 

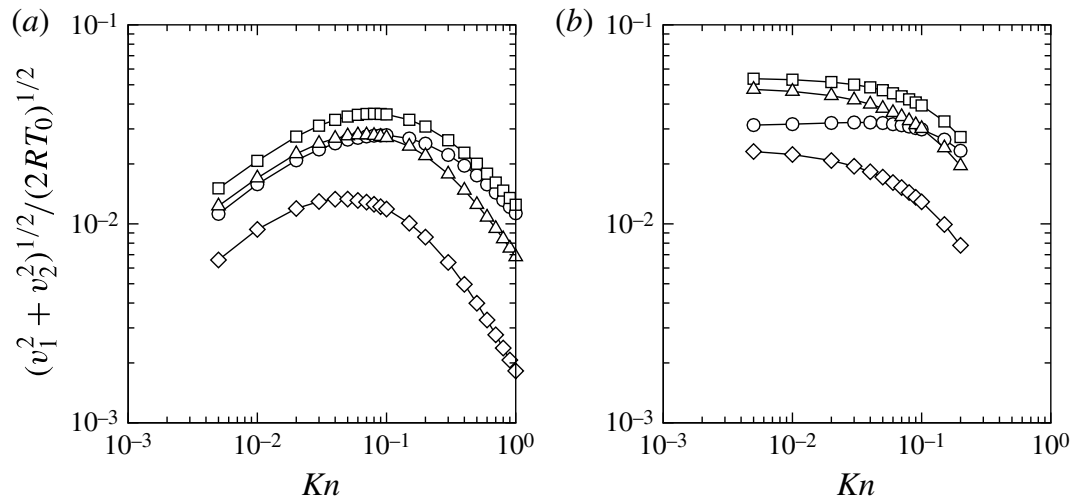

FIGURE 21. Variation of the flow speed with $K n$ of the radiometric flow at various locations in the gas in the case of $T_{w}^{+} / T_{0}=2$ and $T_{w}^{-} / T_{0}=1(L / D=4)$. In $(a)$, the flow speed is evaluated at four locations fixed in the gas: $\circ,\left(X_{1} / D, X_{2} / D\right)=(0.05,0.45) ; \square,(0.05,0.55)$; $\triangle,(-0.05,0.55) ; \diamond,(-0.05,0.45)$. In $(b)$, the flow speed is evaluated at four locations in the gas having the same relative positions to the edge in the scale of the mean free path: $\circ$, $\left(X_{1} / \ell_{0},\left(X_{2}-2 / D\right) / \ell_{0}\right)=(1 / 2 \sqrt{2},-1 / 2 \sqrt{2}) ; \square,(1 / 2 \sqrt{2}, 1 / 2 \sqrt{2}) ; \triangle,(-1 / 2 \sqrt{2}, 1 / 2 \sqrt{2}) ; \diamond$, $(-1 / 2 \sqrt{2},-1 / 2 \sqrt{2})$.

$(-0.05,0.45)$ and $(-0.05,0.55)$, and in $(b)$ those at four locations relatively at the same positions to the edge in the scale of the mean free path, i.e. at $\left(X_{1} / \ell_{0},\left(X_{2}-D / 2\right) / \ell_{0}\right)=(1 / 2 \sqrt{2}, 1 / 2 \sqrt{2}),(1 / 2 \sqrt{2},-1 / 2 \sqrt{2}),(-1 / 2 \sqrt{2}, 1 / 2 \sqrt{2})$ and $(-1 / 2 \sqrt{2},-1 / 2 \sqrt{2})$. Note that, in $(b)$, the distance of the points from the edge is $\ell_{0} / 2$ in the dimensional $\left(X_{1}, X_{2}\right)$ plane.

From figure 21(a), one can see that the flow tends to vanish as the limit $K n \rightarrow 0$ is approached, as in the case of the thermal edge flow. On the other hand, figure 21(b) shows that the flow speed approaches a non-zero limiting value, depending on the relative position to the edge, as $K n$ tends to zero. This means that the flow is not vanishing at the edge in the continuum limit, unlike the case of the thermal edge flow. This conclusion is also consistent with the following numerical result. That is, figure 22 shows the contour plots of the temperature (in $a$ ) and the flow speed (in $b)$ around the edge for four different values of $K n(K n=0.04,0.02,0.01$ and 0.005$)$ shown by using the coordinates scaled by the mean free path (centred at the edge). It is seen from figure 22(a) that the temperature approaches a limiting distribution as $K n$ becomes small. This means that there always exists a finite temperature variation around the edge in the scale of the mean free path, irrespective of the smallness of the global Knudsen number. As a result, a flow with a finite speed is induced near the edge, as indicated by figure $22(b)$; the isolines for the flow speed approach the nonzero lines as $K n \rightarrow 0$. It should be noted, however, that the region with appreciable flow shrinks towards the edge as $K n$ tends to zero. Therefore, the flow remains finite only at the edges and at other points in the gas a state with no flow is established in the continuum limit.

This situation is quite similar to the case of a flow induced by a discontinuous wall temperature that was studied by Aoki et al. (2001b). More precisely, they considered a rarefied gas confined in a square vessel whose left and right halves are maintained at different uniform temperatures and investigated the steady flow induced in the vessel with interest in its behaviour for small Knudsen numbers (based on the reference 

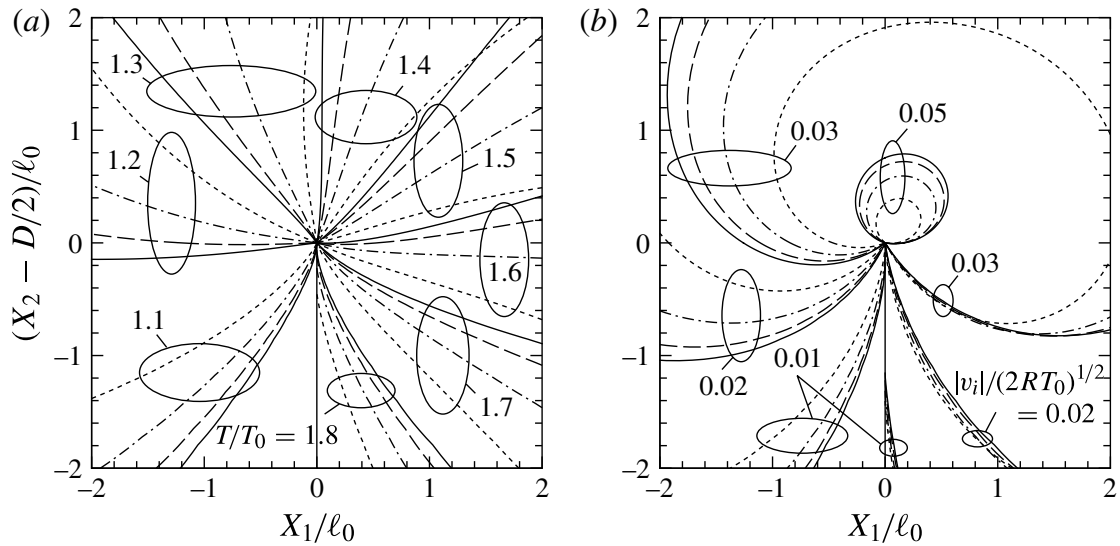

FIGURE 22. Distributions of the temperature $T$ and flow speed $\left|v_{i}\right|=\left(v_{1}^{2}+v_{2}^{2}\right)^{1 / 2}$ near the edge for various $K n\left(T_{w}^{+} / T_{0}=2, T_{w}^{-} / T_{0}=1\right.$ and $\left.L / D=4\right)$. (a) $T,(b)\left|v_{i}\right|$. The solid line represents the isoline for $K n=0.005$, the dashed line for $K n=0.01$, the dash-dotted line for $K n=0.02$ and the dotted line for $K n=0.04$.

mean free path and the size of the vessel). The result is summarized as follows: (i) as the system approaches the continuum limit, the region with appreciable flow speed converges to the point where the wall temperature is discontinuous, and (ii) the flow vanishes at all points except at the points of discontinuity. In the present problem, the plate temperature is discontinuous at the edges, and the flow is driven by the temperature field established there. Therefore, the same singularity also occurs at the edges in the radiometric flow.

\section{Normal stress and the radiometric force}

In this section, we discuss the normal stress on the plate, on the basis of the numerical results, and clarify the cause of the radiometric force.

\subsection{Normal stress}

Figures 23 and 24 show the distributions of the normal stress $p_{11}$ of the thermal edge flow and those of the radiometric flow along several lines parallel to the plate for some Knudsen numbers $(K n=5,0.05$ and 0.01 or 0.02$)$. More precisely, in figure 23 the distributions of the normal stress of the radiometric flow $\left(T_{w}^{+} / T_{0}=2\right.$ and $\left.T_{w}^{-} / T_{0}=1\right)$ along four lines $X_{1} / D=0_{+}, 0.01,0.05$ and 0.1 are compared with the corresponding distributions of the thermal edge flow around a heated plate $\left(T_{w}^{+} / T_{0}=T_{w}^{-} / T_{0}=2\right)$ for $K n=5,0.05$ and 0.01 , and in figure 24 those of the radiometric flow along four lines $X_{1} / D=0_{-},-0.01,-0.05$ and -0.1 are compared with the corresponding ones of the thermal edge flow around a cooled plate $\left(T_{w}^{+} / T_{0}=T_{w}^{-} / T_{0}=0.5\right)$ for $K n=5$, 0.05 and 0.02 . The profiles of $p_{11}$ closely resemble those of the corresponding profiles of the pressure $p$ (figures 16 and 19) for both the thermal edge and radiometric flows. In particular, as for the pressure, a sharp peak develops in the positive- or negative- $p_{11}$ direction near the edge with the decrease of $K n$, depending on whether the corresponding side of the plate is hotter than the environment (figure 23) or colder than it (figure 24).

In order to obtain further information about the peak, let us investigate how the magnitude of the peak varies with $K n$. For this purpose, we introduce the quantity $\tau_{11}$ 

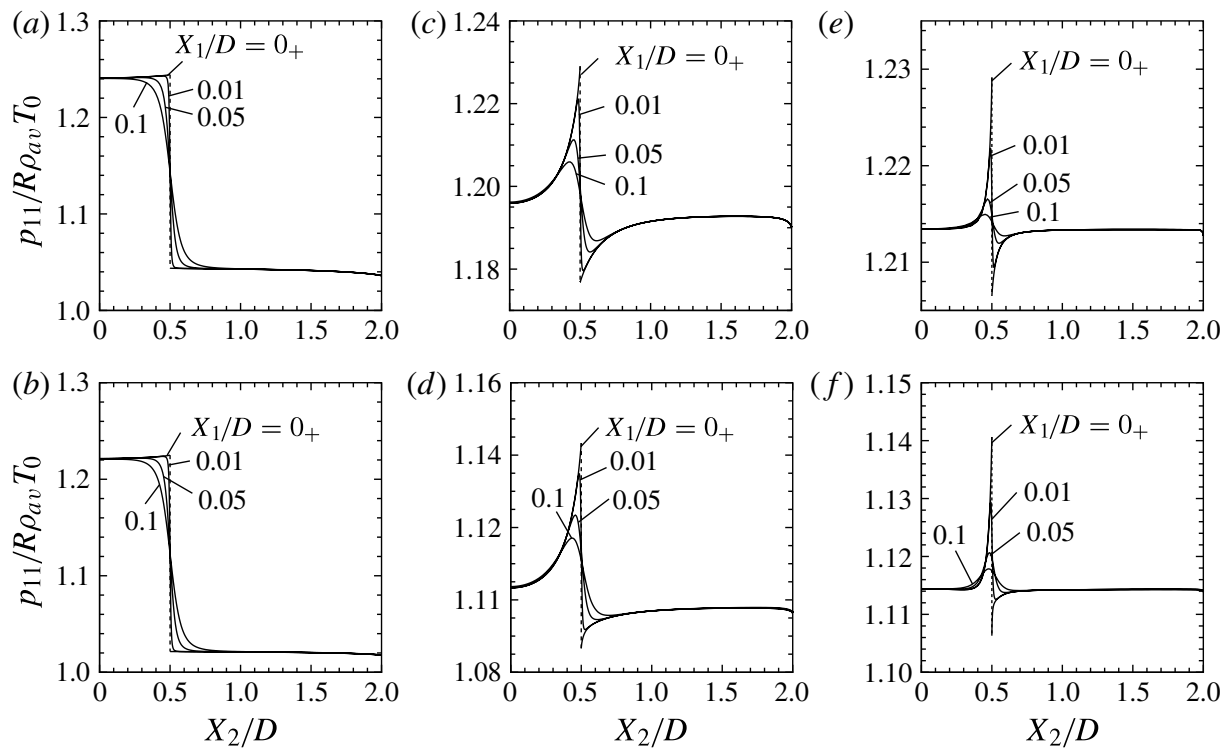

FIgURE 23. Distributions of the stress $p_{11}$ along four lines $X_{1} / D=0_{+}, 0.01,0.05$ and 0.1 close to the plate for various $K n(L / D=4)$. Top row $(a, c, e)$ Thermal edge flow around a heated plate $\left(T_{w}^{+} / T_{0}=T_{w}^{-} / T_{0}=2\right)$; bottom row $(b, d, f)$ radiometric flow $\left(T_{w}^{+} / T_{0}=2\right.$ and $\left.T_{w}^{-} / T_{0}=1\right):(a, b) K n=5 ;(c, d) K n=0.05 ;(e, f) K n=0.01$.
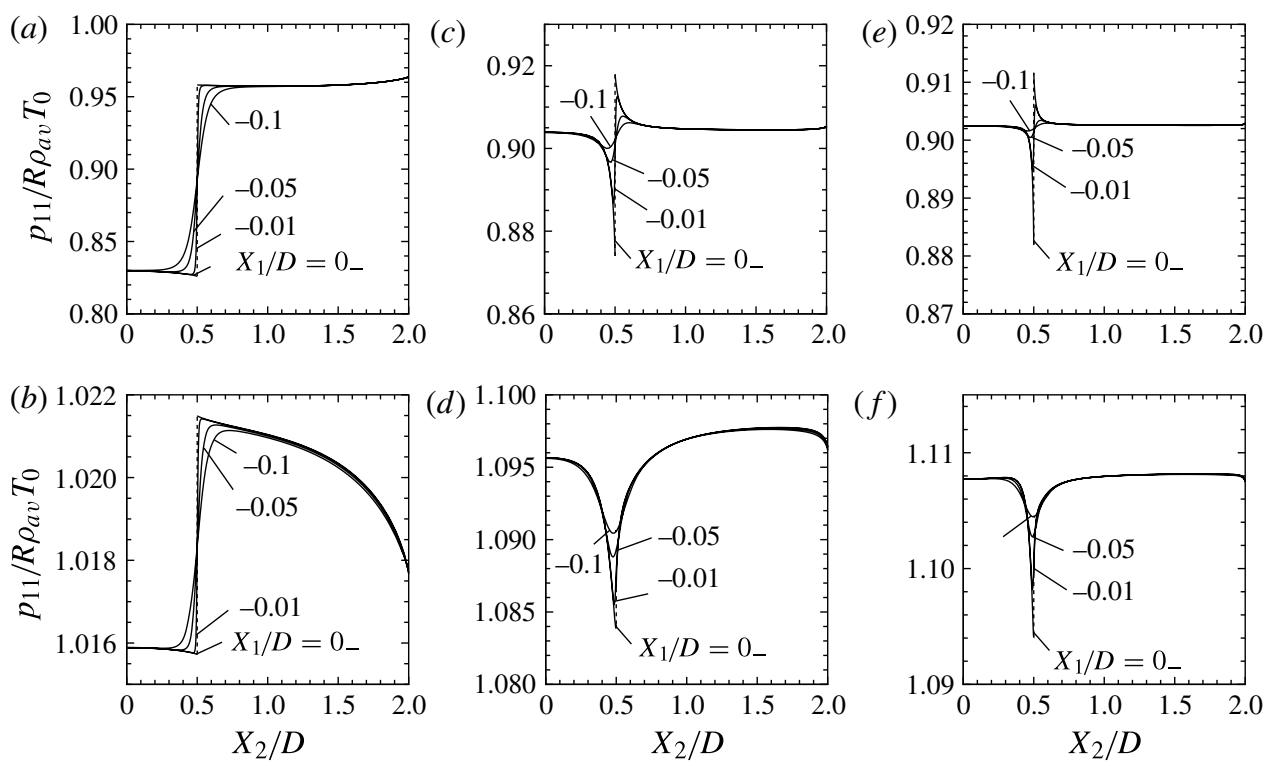

FIGURE 24. Distributions of the stress $p_{11}$ along four lines $X_{1} / D=0_{-},-0.01,-0.05$ and -0.1 close to the plate for various $K n(L / D=4)$. Top row $(a, c, e)$ Thermal edge flow around a cooled plate $\left(T_{w}^{+} / T_{0}=T_{w}^{-} / T_{0}=0.5\right)$; bottom row $(b, d, f)$ radiometric flow $\left(T_{w}^{+} / T_{0}=2\right.$ and $\left.T_{w}^{-} / T_{0}=1\right):(a, b) K n=5 ;(c, d) K n=0.05 ;(e, f) K n=0.02$. 

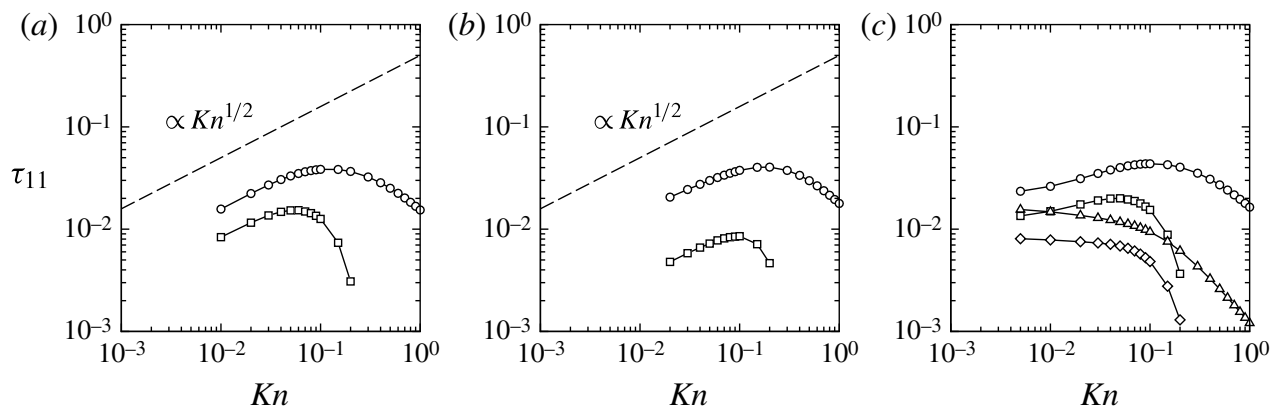

FIgURE 25. $\tau_{11}$ as a function of $K n(L / D=4)$. (a) Thermal edge flow around a heated plate $\left(T_{w}^{+} / T_{0}=T_{w}^{-} / T_{0}=2\right),(b)$ thermal edge flow around a cooled plate $\left(T_{w}^{+} / T_{0}=T_{w}^{-} / T_{0}=0.5\right)$, (c) radiometric flow $\left(T_{w}^{+} / T_{0}=2\right.$ and $\left.T_{w}^{-} / T_{0}=1\right)$. In $(a)$ and $(b)$, o represents the result along $X_{1} / D=0_{+}$and $\square X_{1} / D=K n$. In (c), o represents the result along $X_{1} / D=0_{+}, \square X_{1} / D=K n$, $\triangle X_{1} / D=0_{-}$and $\diamond X_{1} / D=-K n$. In $(a)$ and $(b)$, a dashed line proportional to $K n^{1 / 2}$ is also shown.

as follows: i.e. for a given $x_{1}\left(=X_{1} / D\right)=\alpha_{*}$ (=const)

$$
\tau_{11}\left(\alpha_{*}\right)=\max _{0 \leqslant x_{2} \leqslant 0.5}\left|\hat{p}_{11}\left(x_{1}=\alpha_{*}, x_{2}\right)-\hat{p}_{11}\left(x_{1}=\alpha_{*}, 0\right)\right| .
$$

$\left(R \rho_{a v} T_{0}\right) \tau_{11}$ corresponds to the height of the peak in the profile of $p_{11}$ along a given line $X_{1} / D=\alpha_{*}$, measured from the value at $X_{2} / D=0$ (i.e. the value on the $X_{1}$ axis), and thus $\tau_{11}$ is a measure of the magnitude of the peak. When $\alpha_{*}=0_{+}$or $0_{-}$(i.e. on the plate), $\max _{0 \leqslant x_{2} \leqslant 0.5}$ in (6.1) should be interpreted as $\sup _{0 \leqslant x_{2}<0.5}$ and should be evaluated using the limiting value of $p_{11}$ from below (see the first paragraph of $\S 4.1$ ). It should also be noted that $\tau_{11}$ depends on $K n, T_{w}^{+} / T_{0}, T_{w}^{-} / T_{0}$ and $L / D$ (through the solution) in addition to $\alpha_{*}$ (i.e. the line along which $\tau_{11}$ is evaluated). Here, we shall evaluate $\tau_{11}$ along two lines $X_{1} / D=0_{+}$and $X_{1} / D=K n$ (i.e. $\alpha_{*}=0_{+}$and $K n$ ) in the case of the thermal edge flow and along four lines $X_{1} / D=0_{ \pm}$and $X_{1} / D= \pm K n$ (i.e. $\alpha_{*}=0_{ \pm}$and $\left.\pm K n\right)$ in the case of the radiometric flow. Note that the lines $X_{1} / D= \pm K n$ approach the plate (or the $X_{2}$ axis) with the decrease of $K n$, but the distance from the plate is kept the same in the scale of the mean free path, irrespective of $K n$. Figure 25 shows the variation of $\tau_{11}$ obtained in this way as a function of $K n$; $(a)$ shows the result for the thermal edge flow around a heated plate $\left(T_{w}^{+} / T_{0}=T_{w}^{-} / T_{0}=2\right)$, $(b)$ that for the thermal edge flow around a cooled plate $\left(T_{w}^{+} / T_{0}=T_{w}^{-} / T_{0}=0.5\right)$ and $(c)$ that for the radiometric flow $\left(T_{w}^{+} / T_{0}=2\right.$ and $\left.T_{w}^{-} / T_{0}=1\right)$. In the case of the thermal edge flow ( $a$ and $b$ ), $\tau_{11}$ tends to decay in proportion to the square root of $K n$ as $K n$ is decreased. In the case of the radiometric flow $(c)$, on the other hand, it tends to approach a non-zero limiting value.

The mechanism of the formation of the peak in $p_{11}$ can be understood in the following way. A rarefied gas having a non-uniform temperature distribution is subject to a stress known as the thermal stress (Maxwell 1879; Sone 2002, 2007). Let us consider a (imaginary) plane surface $\mathrm{S}$ in the gas and two points $\mathrm{A}$ and $\mathrm{B}$ on the respective sides of it, located in such a way that the line joining A and B intersects the surface perpendicularly. Let the distance of each point from the surface be, say, one mean free path. The momentum flux across the surface in the direction normal to it is roughly estimated to be the sum of the temperatures at these points, each of which represents the character of the group of molecules impinging on the surface 
from either side. Hence, the normal stress on the surface is (roughly) given by

$$
\text { Stress } \sim T(X-\ell)+T(X+\ell) \sim 2 T+\frac{\mathrm{d}^{2} T}{\mathrm{~d} X^{2}} \ell^{2},
$$

where $X$ is a coordinate normal to the surface and $\ell$ is the mean free path. In the last expression, the second term $\left(\mathrm{d}^{2} T / \mathrm{d} X^{2}\right) \ell^{2}$ corresponds to the thermal stress arising in the gas due to the temperature non-uniformity (Maxwell 1879; Sone 2002, 2007). In this argument we implicitly consider a quiescent gas with temperature variation. Therefore, the density varies, i.e. it is high (low) in the region of low (high) temperature. This density variation reduces the normal stress in (6.2). However, the density variation is determined in such a way that the mass flow $\hat{\rho} \hat{v}_{i}$, the first moment of the velocity distribution function (cf. (2.23)), vanishes. Since the normal stress is the second moment (cf. $(2.30 a)$ ), the effect of the temperature variation, that is, the effect of the difference in the average molecular speed, dominates the effect of the density variation. Therefore, (6.2), where the density variation is neglected, is correct qualitatively.

Now we estimate the magnitude of the $\left(\mathrm{d}^{2} T / \mathrm{d} X^{2}\right) \ell^{2}$ term. Since there is no analytical solution available, we shall exploit here, for simplicity, the solution of the heat-conduction equation. Indeed, it was in this way that Sone \& Yoshimoto (1997) were able to arrive at an estimate of the magnitude of the thermal edge flow. First, we consider the case of the thermal edge flow. The temperature distribution around a semi-infinite plate vertically placed in a large expanse of a gas (extending indefinitely in the downward direction) and having temperature $T_{1}$ is given by $T=T_{1}-a_{*} r^{1 / 2} \cos (\theta / 2)+\cdots$. Here, $r$ is the radial distance from the edge, $\theta$ is the angle measured from the upward direction $(\theta=\pi$ on the right-hand side of the plate and $\theta=-\pi$ on the left-hand side) and $a_{*}$ is a constant ( $a_{*}$ is positive when the plate is heated and is negative when it is cooled). Now we consider three points in the gas, say, $\mathrm{A}, \mathrm{B}$ and $\mathrm{C}$, near the edge, which are aligned along the half-line perpendicular to the plate originating from the edge. The point $\mathrm{A}$ is on the edge and $\mathrm{B}$ and $\mathrm{C}$ are located in such a way that their distances from the edge are $\ell$ and $2 \ell$, respectively. If we denote the temperatures at $\mathrm{A}, \mathrm{B}$ and $\mathrm{C}$ by $T_{A}, T_{B}$ and $T_{C}$, respectively, they are then given by $T_{A}=T_{1}, T_{B}=T_{1}-a_{*} \ell^{1 / 2} / \sqrt{2}$ and $T_{C}=T_{1}-a_{*}(2 \ell)^{1 / 2} / \sqrt{2}$. Thus, by using the centraldifference formula, the second derivative of $T$ with respect to $X$ (the coordinate along the half-line) at $\mathrm{B}$ is evaluated as $\partial^{2} T / \partial X^{2} \sim\left(T_{A}-2 T_{B}+T_{C}\right) / \ell^{2} \sim(\sqrt{2}-1) a_{*} \ell^{-3 / 2}$, giving the result that the thermal stress is proportional to $a_{*} \sqrt{\ell}$. It should be noted that $\partial^{2} T / \partial X^{2}$ decreases with distance in the downward direction along the plate, as can be seen from the isothermal lines in figure 6 that are more parallel to the plate and more equally spaced near the centre than near the edge. Therefore, the thermal stress is appreciable only near the edge. As a consequence, the thermal stress produces, in $p_{11}$, a rise towards the edge along the plate when the plate is heated and a fall when the plate is cooled. Moreover, its height (or depth) is of the order of the square root of the mean free path and this is perfectly consistent with our numerical result (figures $25 a$ and 25b). In the case of the radiometric flow, we can argue in the same way except that the corresponding temperature distribution is now given by $T=\left(T_{0}+T_{1}\right) / 2+\left(T_{1}-T_{0}\right) \theta / 2 \pi-b_{*} r^{1 / 2} \cos (\theta / 2)+\cdots$, where $T_{0}$ and $T_{1}\left(>T_{0}\right)$ are, respectively, the temperatures on the colder and the hotter side of the plate and $b_{*}$ is a constant. A parallel calculation shows that $\left(\partial^{2} T / \partial X^{2}\right) \ell^{2} \sim\left(T_{1}-T_{0}\right) / 4$ on the hotter side and $\left(\partial^{2} T / \partial X^{2}\right) \ell^{2} \sim-\left(T_{1}-T_{0}\right) / 4$ on the colder side. Thus, along the plate, there will be a rise and a fall in $p_{11}$ towards the edge on the hotter side and the colder (or unheated) side of the plate, respectively. Since the magnitude of the thermal stress 
Rarefied gas flow around a sharp edge
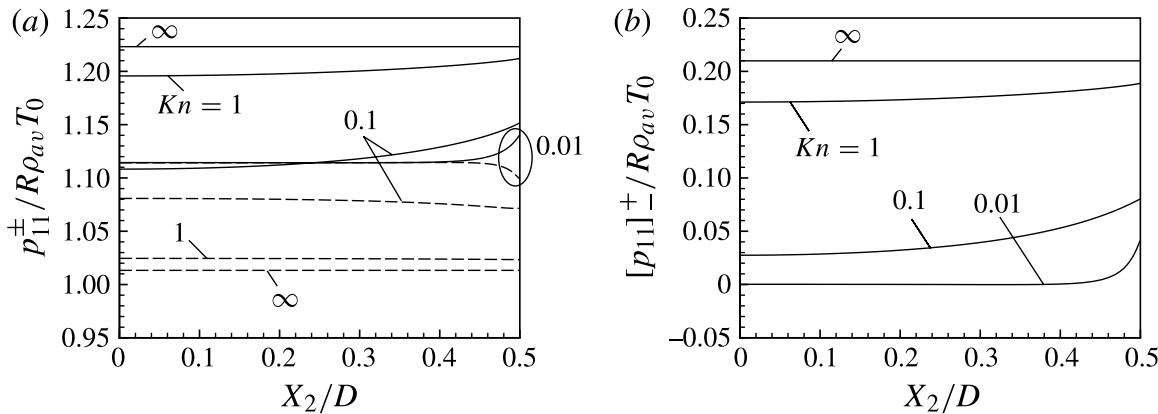

FIGURE 26. (a) Distributions of the normal stress, $p_{11}^{+}=\left.p_{11}\left(X_{1}, X_{2}\right)\right|_{X_{1} / D=0_{+}}$and $p_{11}^{-}=\left.p_{11}\left(X_{1}, X_{2}\right)\right|_{X_{1} / D=0_{-}}$, along each side of the plate and $(b)$ the difference $\left[p_{11}\right]_{-}^{+}=p_{11}^{+}-p_{11}^{-}$ in the radiometric flow $\left(T_{w}^{+} / T_{0}=2, T_{w}^{-} / T_{0}=1\right.$ and $\left.L / D=4\right)$. In $(a)$, the solid line represents $p_{11}^{+}$and the dashed line $p_{11}^{-}$.

is independent of the mean free path, it develops into a 'peak' localised around the edge with the decrease of $K n$. Again, this is consistent with our numerical result (figure 25c).

In summary, from the above discussion and the numerical result to support it, it is almost certain that the peak in $p_{11}$ arising near the edge is caused by the effect of the thermal stress. In the case of the thermal edge flow (or the case in which temperatures on both sides of the plate are the same), the peak decays in proportion to the square root of the mean free path, while in the case of the radiometric flow (or the case in which temperatures on both sides are different), it is independent of the mean free path (and thus does not vanish at the edge in the continuum limit).

\subsection{Origin of the force acting on the plate in the radiometric flow}

Now we turn to the question of the cause of the radiometric force. Figure 26 illustrates how the distribution of the normal stress on each side of the plate varies with $K n$ in the case of the radiometric flow $\left(T_{w}^{+} / T_{0}=2\right.$ and $\left.T_{w}^{-} / T_{0}=1\right)$; (a) shows the actual distributions of the normal stresses $p_{11}^{+}=\left.p_{11}\left(X_{1}, X_{2}\right)\right|_{X_{1} / D=0_{+}}$ and $p_{11}^{-}=\left.p_{11}\left(X_{1}, X_{2}\right)\right|_{X_{1} / D=0_{-}}$along each side of the plate and $(b)$ the difference $\left[p_{11}\right]_{-}^{+}=p_{11}^{+}-p_{11}^{-}$. In the free molecular flow $(K n \rightarrow \infty), p_{11}^{+}$and $p_{11}^{-}$are uniform along the plate. For large $K n$, there is a significant difference between $p_{11}^{+}$and $p_{11}^{-}$ in the entire range of $X_{2} / D$ along the plate (this corresponds to the radiometric force at a large $K n$ ). As $K n$ becomes small, the difference between $p_{11}^{+}$and $p_{11}^{-}$tends to vanish, except near the edge $\left(X_{2} / D \sim 0.5\right)$. Therefore, the plate experiences a net force perpendicular to it only in the edge region and no force on the other parts. On the other hand, from the result of the preceding subsection, this difference between $p_{11}^{+}$and $p_{11}^{-}$near the edge comes from the thermal stress, which has different signs on each side. Thus, the radiometric force is caused by the thermal stress.

Finally, we show in figure 27 the variation of the total force $(F, 0,0)$ acting on the plate (per unit width in $X_{3}$ ) as a function of $K n\left(T_{w}^{+} / T_{0}=2\right.$ and $\left.T_{w}^{-} / T_{0}=1\right)$. Here, $F$ is given by

$$
\begin{aligned}
F & =-2 \int_{0}^{D / 2}\left[p_{11}\right]_{-}^{+} \mathrm{d} X_{2} \\
& =-2 \int_{0}^{D / 2}\left[\left.p_{11}\left(X_{1}, X_{2}\right)\right|_{X_{1} / D=0_{+}}-\left.p_{11}\left(X_{1}, X_{2}\right)\right|_{X_{1} / D=0_{-}}\right] \mathrm{d} X_{2}
\end{aligned}
$$




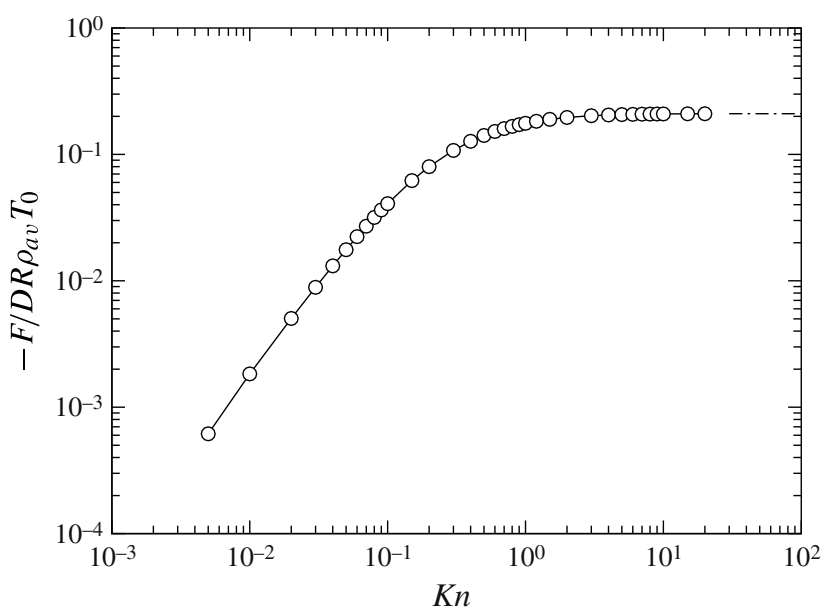

FIGURE 27. Radiometric force $F$ versus $K n$ in the case of $T_{w}^{+} / T_{0}=2$ and $T_{w}^{-} / T_{0}=1$ $(L / D=4)$. The symbols represents the numerical results which are connected by solid lines. The dash-dotted line indicates the limiting value $F / D R \rho_{a v} T_{0} \rightarrow-0.20986$ as $K n \rightarrow \infty$.

$\begin{array}{lccccc}K n & -F / D R \rho_{a v} T_{0} & K n & -F / D R \rho_{a v} T_{0} & K n & -F / D R \rho_{a v} T_{0} \\ 0.005 & 0.00062 & 0.2 & 0.08000 & 3 & 0.20226 \\ 0.01 & 0.00183 & 0.3 & 0.10753 & 4 & 0.20504 \\ 0.02 & 0.00503 & 0.4 & 0.12698 & 5 & 0.20653 \\ 0.03 & 0.00888 & 0.5 & 0.14113 & 6 & 0.20744 \\ 0.04 & 0.01313 & 0.6 & 0.15182 & 7 & 0.20802 \\ 0.05 & 0.01761 & 0.7 & 0.16007 & 8 & 0.20842 \\ 0.06 & 0.02235 & 0.8 & 0.16660 & 9 & 0.20871 \\ 0.07 & 0.02703 & 0.9 & 0.17186 & 10 & 0.20892 \\ 0.08 & 0.03171 & 1 & 0.17617 & 15 & 0.20946 \\ 0.09 & 0.03634 & 1.2 & 0.18278 & 20 & 0.20966 \\ 0.1 & 0.04078 & 1.5 & 0.18947 & \infty & 0.20986 \\ 0.15 & 0.06204 & 2 & 0.19606 & & \end{array}$

TABLE 1 . Radiometric force $F$ versus $K n$ for $T_{w}^{+} / T_{0}=2$ and $T_{w}^{-} / T_{0}=1(L / D=4)$.

The corresponding values are given in table $1 .-F / D R \rho_{a v} T_{0}$ increases monotonically with $K n$. For each set of values $T_{w}^{+} / T_{0}, T_{w}^{-} / T_{0}$ and $L / D$, the force has a limiting value as $K n \rightarrow \infty$. In the present case of $T_{w}^{+} / T_{0}=2, T_{w}^{-} / T_{0}=1$ and $L / D=4, F / D R \rho_{a v} T_{0} \rightarrow-0.20986$ as $K n \rightarrow \infty$. It should be noted, however, that the dimensional force $-F$ decreases as $K n \rightarrow \infty$ because $D \rho_{a v}$ generally decreases with the increase of $K n$.

\section{Concluding remarks}

In this paper, we have considered a rarefied gas flow induced around the sharp edges of a flat plate by the effect of a temperature field. Typical examples of such flows are the thermal edge flow and the radiometric flow. The former is induced when the plate is uniformly heated (or cooled), and the latter is induced when the two sides of the plate are maintained at different temperatures. In this paper, we have investigated these 
flows numerically on the basis of the BGK model Boltzmann equation and the diffuse reflection boundary condition.

After the formulation of the problem (\$2), a numerical method that is able to give a correct description of the discontinuity contained in the velocity distribution function was introduced ( $\$ 3$ and appendix A). Then, we have carried out an extensive numerical analysis on the radiometric and the thermal edge flows for a wide range of the Knudsen number. As a result, the following results were obtained. First, we have clarified the existence of the similarity between the thermal edge flow and the radiometric flow, particularly in the flow structure near the edge. In addition, we have developed, on the basis of this analogy, an understanding of the mechanism of the radiometric flow for the infinitely thin plate $(\$ 4)$. Second, we have investigated the limiting behaviour of the flows as the Knudsen number tends to zero. The result shows that there is an important difference between the thermal edge flow and the radiometric flow in the continuum limit, despite the above similarity. That is, the former vanishes everywhere in the gas, whereas the latter does so except at the edges (\$5). Finally, we have clarified the structure of the normal stress (on the plate), particularly that near the plate. We have shown that the abrupt temperature variation near the edge gives rise to a strong thermal stress there and this thermal stress causes a net force on the plate when the temperatures on both sides are different from each other ( $§ 6)$.

The flows studied here are essentially caused by abrupt temperature variation near the edges, and the region where the flow is appreciable shrinks as the Knudsen number decreases. Therefore, it is essential to use a numerical method capable of resolving overall tiny flow structures as well as abrupt change near the edges. For this purpose, we used the finite-difference method, rather than the conventional DSMC method. The latter method would need a huge number of particles and cells as well as many averaging steps to meet our requirements. We used the BGK model instead of the original Boltzmann equation because the required high resolution is still not possible for the latter. It is well known that, for the BGK model, the Prandtl number becomes unity, not around $2 / 3$ as given by the Boltzmann equation for most of intermolecular potentials. However, some existing results show that, in the case of thermally driven flows, the BGK results show good agreement with the Boltzmann results for hardsphere molecules (see e.g. Ohwada, Sone \& Aoki 1989b; Aoki, Degond \& Mieussens 2009). In these comparisons, the mean free path of the BGK model is converted to that of the Boltzmann equation in such a way that the thermal conductivity is a common quantity. One can expect similar agreements between the BGK model and more sophisticated models, such as the ellipsoidal statistical (ES) model. In fact, both models give the same results for the linearized thermal transpiration through a channel (Takata, Funagane \& Aoki 2010).

In the present study, we have considered a plate without thickness, which is an idealised situation. However, even if the plate is thicker and with round edges, and even if the temperature change at the edges is not exactly discontinuous, we may expect qualitatively the same behaviour of the gas. Indeed, it was found that the thermal edge flow is induced even when the edge is quite round (Aoki, Sone \& Waniguchi 1998). Therefore, the features of the flow field obtained here are unlikely to be restricted to the case of a flat plate without thickness and are likely to represent the features in more general situations. In the present two-dimensional setting, when the gas extends to infinity (instead of being enclosed in a container), we cannot control the temperature at infinity. Therefore, the problem may be trivial: for instance, in the case of the thermal edge flow, the temperature at infinity should be the same as that of the plate, so that no flow is induced in the gas. However, when we consider a 
three-dimensional problem, say with a rectangular plate, the case in which the gas extends to infinity makes sense, and we may expect that similar flows are induced by the same mechanism as discussed in this paper.

\section{Acknowledgements}

This work was partially supported by a grant-in-aid for scientific research from JSPS (No. 21656026).

\section{Appendix A. Outline of the numerical analysis}

(i) For the numerical analysis, we introduce the polar coordinates $\left(\zeta, \theta_{\zeta}\right)$ in the $\left(\zeta_{1}, \zeta_{2}\right)$ plane defined by

$$
\zeta_{1}=\zeta \cos \theta_{\zeta}, \quad \zeta_{2}=\zeta \sin \theta_{\zeta}
$$

$\left(0 \leqslant \zeta<\infty ;-\pi \leqslant \theta_{\zeta} \leqslant \pi\right)$, and regard $\Phi$ as a function of $\left(x_{1}, x_{2}, \zeta, \theta_{\zeta}\right)$ $\left(\Phi\left(x_{1}, x_{2}, \zeta, \pi\right)=\Phi\left(x_{1}, x_{2}, \zeta,-\pi\right)\right)$. Since the equations and boundary conditions for the new $\Phi$ are readily obtained from (2.21)-(2.29), we omit the explicit form.

(ii) We restrict the range of $\zeta$ to $0 \leqslant \zeta \leqslant Z$, where $Z$ is chosen in such a way that $\Phi$ is negligibly small around $\zeta \simeq Z$. We obtain the discrete solution $\Phi_{\sharp}$ of $\Phi$ at the lattice points $\left(x_{1}^{(i)}, x_{2}^{(j)}, \zeta^{(k)}, \theta_{\zeta}^{(l)}\right)$ in the $\left(x_{1}, x_{2}, \zeta, \theta_{\zeta}\right)$ space as the limit of the sequence $\left\{\Phi_{\sharp}^{(n)}\right\}(n=0,1, \ldots)$ constructed by a finite-difference equation corresponding to (2.21) that gives the relation between $\Phi_{\sharp}^{(n)}$ and $\Phi_{\sharp}^{(n-1)}$, where the superscript $(n)$ denotes the value at the $n$th step of iteration. Let us assume that $\Phi_{\sharp}^{(n-1)}$ is known. For each $0 \leqslant$ $\theta_{\zeta}^{(l)}<\pi / 2$ (or for each $-\pi / 2<\theta_{\zeta}^{(l)}<0$ ), $\Phi_{\sharp}^{(n)}$ is determined from $x_{1}=-\hat{L} / 2$ to $\hat{L} / 2$ and from $x_{2}=0$ to $\hat{L} / 2$ (or from $x_{2}=\hat{L} / 2$ to 0 ) by using the finite-difference equation, the boundary condition (2.27) at $x_{1}=-\hat{L} / 2$, the boundary condition (2.25) (with upper sign) on the right-hand side of the plate and the specular reflection condition (2.29) at $x_{2}=$ 0 (or the boundary condition (2.27) at $x_{2}=\hat{L} / 2$ ) for all $\zeta^{(k)}$ (see figure 28a). Similarly, for each $\pi / 2<\theta_{\zeta}^{(l)} \leqslant \pi$ (or for each $-\pi \leqslant \theta_{\zeta}^{(l)}<-\pi / 2$ ), $\Phi_{\sharp}^{(n)}$ is determined from $x_{1}=\hat{L} / 2$ to $-\hat{L} / 2$ and from $x_{2}=0$ to $\hat{L} / 2$ (or from $x_{2}=\hat{L} / 2$ to 0 ) by using the finite-difference equation, the boundary condition (2.27) at $x_{1}=\hat{L} / 2$, the boundary condition (2.25) (with lower sign) on the left-hand side of the plate and the specular reflection condition (2.29) at $x_{2}=0$ (or the boundary condition $\left(2.27\right.$ ) at $x_{2}=\hat{L} / 2$ ) for all $\zeta^{(k)}$ (see figure $28 b$ ). For $\theta_{\zeta}^{(l)}=\pi / 2\left(\right.$ or for $\theta_{\zeta}^{(l)}=-\pi / 2$ ), $\Phi_{\sharp}^{(n)}$ is determined from $x_{2}=0$ to $\hat{L} / 2$ (or from $x_{2}=\hat{L} / 2$ to 0$)$ using the finite-difference equation and the specular reflection condition (2.29) at $x_{2}=0$ (or the boundary condition (2.27) at $x_{2}=\hat{L} / 2$ ) for all $\zeta^{(k)}$.

(iii) As explained in $\S 3.1$, the velocity distribution function is discontinuous in the gas. Here, we neglect the discontinuities caused by the corners and concentrate on those caused by the edges (see the third paragraph of $\S 3.1$ ). Then, for a given $\left(\zeta^{(k)}, \theta_{\zeta}^{(l)}\right)=(\alpha, \beta)$ with $\alpha>0$ and $0 \leqslant \beta \leqslant \pi / 2$ (or $-\pi / 2 \leqslant \beta<0$ ), $\Phi$ is discontinuous along the lines $x_{2}=(\tan \beta) x_{1}+1 / 2$ (the line between the regions I and II in figure $28 a$ ) and $x_{2}=(\tan \beta) x_{1}-1 / 2$ (the line between the regions II and IV in figure 28a) (or along the line $x_{2}=(\tan \beta) x_{1}+1 / 2$, i.e. the line between the regions II and III in figure $28 a$ ). The application of the finite-difference expressions for $\partial \Phi / \partial x_{1}$ and $\partial \Phi / \partial x_{2}$ across the discontinuity line introduces a significant error. Therefore, a local correction is required to the finite-difference procedure explained in (ii) near the discontinuity line. First, we decompose the domain into smaller regions 
(a)
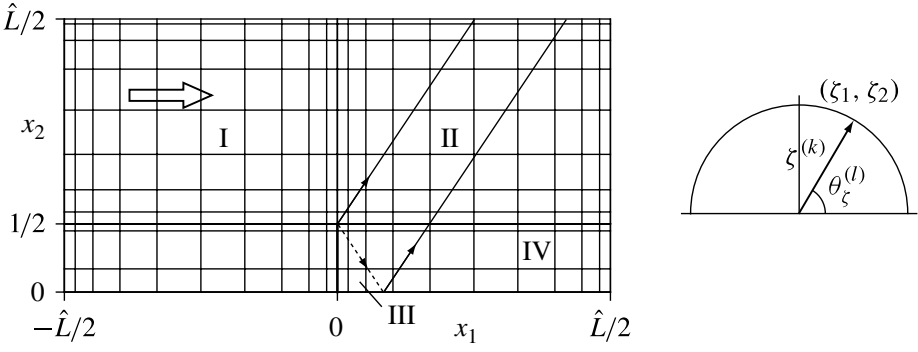

(b)
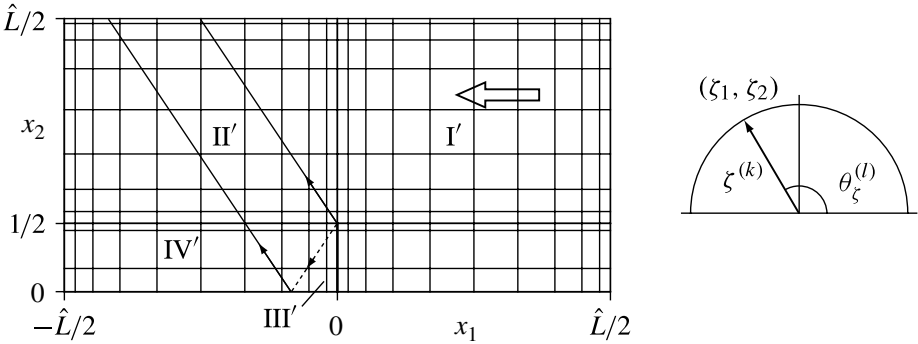

FIGURE 28. Schematic illustration of the process of numerical analysis. (a) $\left|\theta_{\zeta}^{(l)}\right|<\pi / 2$,

$$
\text { (b) } \pi / 2<\left|\theta_{\zeta}^{(l)}\right| \leqslant \pi \text {. }
$$

I, II + III and IV (or into I + II + IV and III). Since there is no discontinuity in each region, we can use the same finite-difference equation as in (ii) in each region. On the other hand, since the regions are now separated by the line of discontinuity, we need the limiting value of $\Phi$ from the right along the discontinuity line as the boundary condition for the regions II + III and IV (or the region I + II + IV) in the process of determining $\Phi_{\sharp}^{(n)}$ from $x_{1}=-\hat{L} / 2$ to $\hat{L} / 2$ in (ii). We obtain this limiting value separately with the aid of another finite-difference equation for (2.21) along (the right-hand side of) the discontinuity line. The case of $\left(\zeta^{(k)}, \theta_{\zeta}^{(l)}\right)=\left(\alpha^{\prime}, \beta^{\prime}\right)$ with $\alpha^{\prime}>0$ and $\pi / 2<\beta^{\prime} \leqslant \pi$ (or $-\pi \leqslant \beta^{\prime}<-\pi / 2$ ) is treated in a similar way. In this case, we divide the original domain into the regions $\mathrm{I}^{\prime}, \mathrm{II}^{\prime}+\mathrm{III}^{\prime}$ and $\mathrm{IV}^{\prime}$ by the discontinuity lines $x_{2}=\left(\tan \beta^{\prime}\right) x_{1}+1 / 2$ (the line between the regions $\mathrm{I}^{\prime}$ and $\mathrm{II}^{\prime}$ in figure $28 b$ ) and $x_{2}=\left(\tan \beta^{\prime}\right) x_{1}-1 / 2$ (the line between the regions $\mathrm{II}^{\prime}$ and $\mathrm{IV}^{\prime}$ in figure $28 b$ ) (or into the regions $\mathrm{I}^{\prime}+\mathrm{II}^{\prime}+\mathrm{IV}^{\prime}$ and $\mathrm{III}^{\prime}$ by the discontinuity line $x_{2}=\left(\tan \beta^{\prime}\right) x_{1}+1 / 2$, i.e. the

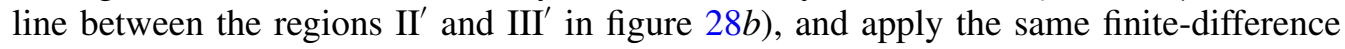
equation as in (ii) in each region. At this point, we need the limiting value of $\Phi$ from the left along the discontinuity line as the boundary condition for the regions II $^{\prime}+$ III' $^{\prime}$ and $\mathrm{IV}^{\prime}$ (or for the region $\mathrm{I}^{\prime}+\mathrm{II}^{\prime}+\mathrm{IV}^{\prime}$ ) for the process of determining $\Phi_{\sharp}^{(n)}$ from $x_{1}=\hat{L} / 2$ to $-\hat{L} / 2$. This limiting value is obtained separately with the aid of another finite-difference equation for (2.21) along (the left-hand side of) the discontinuity line.

\section{Appendix B. Accuracy of the numerical computation}

The grid systems were chosen carefully and various tests were performed for both the thermal edge flow and the radiometric flow to confirm the accuracy. To save space, we confine our attention to the case of the radiometric flow, omitting the details for the thermal edge flow. In general, it is easier to attain higher accuracy in the thermal edge flow than in the radiometric flow. Hereafter, we assume $\hat{L}=4$ (see $\S 3.2$ ). 
The domain $-2 \leqslant x_{1} \leqslant 2$ and $0 \leqslant x_{2} \leqslant 2$ is subdivided into $2 M \times M$ rectangular domains $\left(\left(x_{1}\right.\right.$-direction $) \times\left(x_{2}\right.$-direction $\left.)\right)$ by the lattice lines. Here, the distribution of lines $x_{1}=$ const is symmetric with respect to $x_{1}=0$ (the $x_{2}$ axis). The lattice lines are dense near $x_{1}=-2$ and 2 and $x_{2}=0$ and 2 as well as around $x_{1}=0$ and $x_{2}=0.5$, and the smallest lattice interval occurs between $x_{1}=0$ and the neighbouring line $x_{1}=$ const with respect to the $x_{1}$ direction and between $x_{2}=0.5$ and the neighbouring line $x_{2}=\operatorname{const}(<0.5)$ with respect to the $x_{2}$ direction. The actual value of $M$ is given below, together with some reference values for the lattice intervals. For the discretisation with respect to $\left(\zeta, \theta_{\zeta}\right)$, the domain $0 \leqslant \zeta \leqslant Z(=8.58)$ and $-\pi \leqslant \theta_{\zeta} \leqslant \pi$ is subdivided into $M_{\zeta} \times M_{\theta_{\zeta}}\left(M_{\zeta}=72\right.$ and $\left.M_{\theta_{\zeta}}=480\right)$ rectangular domains ( $(\zeta-$ direction $) \times\left(\theta_{\zeta}\right.$-direction $\left.)\right)$ by the lattice lines. The distribution of lines $\theta_{\zeta}=$ const is uniform, whereas that of lines $\zeta=$ const is non-uniform. It is finer near $\zeta=0$ and coarser near $\zeta=Z$, and the smallest (largest) lattice interval is $2.02 \times 10^{-3}(0.349)$ at $\zeta=0($ at $\zeta=Z$ ).

The choice of $M$ is summarized as follows. $M=320$ for $0.005 \leqslant K n \leqslant 0.05$ and $M=160$ for $0.06 \leqslant K n \leqslant 20$. The smallest (largest) lattice interval is $5.7 \times 10^{-6}$ $\left(3.1 \times 10^{-2}\right)$ for $K n=0.005,1.4 \times 10^{-5}\left(2.8 \times 10^{-2}\right)$ for $K n=0.01,3.4 \times 10^{-5}$ $\left(2.5 \times 10^{-2}\right)$ for $0.02 \leqslant K n \leqslant 0.05,8.4 \times 10^{-4}\left(3.2 \times 10^{-2}\right)$ for $0.06 \leqslant K n \leqslant 1$ and $3.7 \times 10^{-3}\left(2.1 \times 10^{-2}\right)$ for $1.2 \leqslant K n \leqslant 20$ in both the $x_{1}$ and $x_{2}$ directions.

In order to estimate the accuracy of the present computation, we checked the changes in the macroscopic variables when the number of lattice lines is almost halved or doubled in the $\left(x_{1}, x_{2}\right)$ plane or in the $\left(\zeta, \theta_{\zeta}\right)$ plane, by removing every other lattice line or by adding new lattice lines between two lines of the standard lattice system mentioned above. Let us denote by $\Delta_{x}^{(1 / 2)}(h)$ and $\Delta_{x}^{(2)}(h)$ the variations in $h\left(h=\hat{\rho}, \hat{v}_{1}\right.$, etc.) when the value of $M$ is halved and doubled, respectively, and by $\Delta_{\zeta}^{(1 / 2)}(h)$ and $\Delta_{\zeta}^{(2)}(h)$ the variations in $h$ when $M_{\zeta}$ and $M_{\theta_{\zeta}}$ are simultaneously halved and doubled, respectively. For $K n=5,0.5$ and 0.05 these are summarized as follows:

$$
\begin{aligned}
& \Delta_{x}^{(2)}(h)<4.4 \times 10^{-5}(h=\hat{\rho}, \hat{T}, \hat{p}), \Delta_{x}^{(2)}\left(\hat{v}_{1}\right)<7.1 \times 10^{-6}, \\
& \Delta_{x}^{(2)}\left(\hat{v}_{2}\right)<9.5 \times 10^{-6}, \\
& \Delta_{\zeta}^{(2)}(h)<1.5 \times 10^{-5}(h=\hat{\rho}, \hat{T}, \hat{p}), \Delta_{\zeta}^{(2)}\left(\hat{v}_{1}\right)<4.4 \times 10^{-6}, \\
& \Delta_{\zeta}^{(2)}\left(\hat{v}_{2}\right)<5.1 \times 10^{-6}, \quad(\text { for } K n=5), \\
& \Delta_{x}^{(2)}(h)<8.4 \times 10^{-5}(h=\hat{\rho}, \hat{T}, \hat{p}), \Delta_{x}^{(2)}\left(\hat{v}_{1}\right)<2.1 \times 10^{-5}, \\
& \Delta_{x}^{(2)}\left(\hat{v}_{2}\right)<3.3 \times 10^{-5}, \quad \\
& \Delta_{\zeta}^{(2)}(h)<9.0 \times 10^{-5}(h=\hat{\rho}, \hat{T}, \hat{p}), \Delta_{\zeta}^{(2)}\left(\hat{v}_{1}\right)<4.1 \times 10^{-6}, \\
& \Delta_{\zeta}^{(2)}\left(\hat{v}_{2}\right)<1.7 \times 10^{-5}, \quad(\text { for } K n=0.5), \\
& \Delta_{x}^{(1 / 2)}(h)<7.1 \times 10^{-4}(h=\hat{\rho}, \hat{T}, \hat{p}), \Delta_{x}^{(1 / 2)}\left(\hat{v}_{1}\right)<2.2 \times 10^{-4}, \\
& \Delta_{x}^{(1 / 2)}\left(\hat{v}_{2}\right)<1.0 \times 10^{-4}, \quad \\
& \Delta_{\zeta}^{(1 / 2)}(h)<2.1 \times 10^{-4}(h=\hat{\rho}, \hat{T}, \hat{p}), \Delta_{\zeta}^{(1 / 2)}\left(\hat{v}_{1}\right)<1.0 \times 10^{-5}, \\
& \Delta_{\zeta}^{(1 / 2)}\left(\hat{v}_{2}\right)<9.3 \times 10^{-5}, \quad(\text { for } K n=0.05) .
\end{aligned}
$$




\section{Rarefied gas flow around a sharp edge}

Another measure of accuracy is obtained by checking the conservation laws. Let us consider the closed domain (or the control surface) $0 \leqslant x_{1} \leqslant r_{1}$ and $r_{2}^{*} \leqslant x_{2} \leqslant r_{2}$ in the $\left(x_{1}, x_{2}\right)$ plane, whose left side is on the $x_{2}$ axis. Let us call the left side $\left(x_{1}=0\right)$ of the domain side I, the right side $\left(x_{1}=r_{1}\right)$ side II, the bottom side $\left(x_{2}=r_{2}^{*}\right)$ side III and the top side $\left(x_{2}=r_{2}\right)$ side IV. If we denote the mass, momentum in the $X_{1}$ direction, momentum in the $X_{2}$ direction and energy flowing out of the domain through the side $J\left(J=\mathrm{I}\right.$, II, III or IV) (per unit time and unit width in $\left.X_{3}\right)$ by $\rho_{a v}\left(2 R T_{0}\right)^{1 / 2} D \mathscr{M}_{J}, R \rho_{a v} T_{0} D \mathscr{P}_{1 J}, R \rho_{a v} T_{0} D \mathscr{P}_{2 J}$ and $\left(\rho_{a v} / 2\right)\left(2 R T_{0}\right)^{3 / 2} D \mathscr{E}_{J}$, respectively, the sums $\mathscr{M}=\sum_{J=I}^{I V} \mathscr{M}_{J}, \mathscr{P}_{1}=\sum_{J=I}^{I V} \mathscr{P}_{1 J}, \mathscr{P}_{2}=\sum_{J=I}^{I V} \mathscr{P}_{2 J}$ and $\mathscr{E}=\sum_{J=I}^{I V} \mathscr{E}_{J}$ should theoretically vanish. However, in reality, they do not vanish exactly due to the numerical error, and the deviation from zero can be used as a measure of accuracy of the present computation. Some examples of these are:

$$
\begin{aligned}
& |\mathscr{M}|=2.1 \times 10^{-6}\left(\mathscr{M}_{I V}=-3.7 \times 10^{-4}\right), \\
& \left|\mathscr{P}_{1}\right|=1.3 \times 10^{-4}\left(\mathscr{P}_{1 I}=-2.3 \times 10^{-1}\right), \\
& \left|\mathscr{P}_{2}\right|=8.1 \times 10^{-5}\left(\mathscr{P}_{2 I I I}=-2.1 \times 10^{-1}\right), \\
& |\mathscr{E}|=-3.5 \times 10^{-4}\left(\mathscr{E}_{I}=-5.4 \times 10^{-2}\right), \\
& \quad\left(\text { for } K n=5 \text { and }\left(r_{1}, r_{2}^{*}, r_{2}\right)=(0.189,0.398,0.602)\right), \\
& |\mathscr{M}|=4.3 \times 10^{-6}\left(\mathscr{M}_{I I I}=2.1 \times 10^{-3}\right), \\
& \left|\mathscr{P}_{1}\right|=2.3 \times 10^{-5}\left(\mathscr{P}_{1 I}=-2.0 \times 10^{-1}\right), \\
& \left|\mathscr{P}_{2}\right|=1.2 \times 10^{-5}\left(\mathscr{P}_{2 I I I}=-2.3 \times 10^{-1}\right), \\
& |\mathscr{E}|=7.2 \times 10^{-5}\left(\mathscr{E}_{I}=-3.2 \times 10^{-2}\right), \\
& \quad\left(\text { for } K n=0.5 \text { and }\left(r_{1}, r_{2}^{*}, r_{2}\right)=(0.203,0.411,0.589)\right), \\
& |\mathscr{M}|=1.8 \times 10^{-6}\left(\mathscr{M}_{I V}=3.2 \times 10^{-3}\right), \\
& \left|\mathscr{P}_{1}\right|=1.7 \times 10^{-6}\left(\mathscr{P}_{1 I}=-2.0 \times 10^{-1}\right), \\
& \left|\mathscr{P}_{2}\right|=8.5 \times 10^{-7}\left(\mathscr{P}_{2 I I I}=-2.2 \times 10^{-1}\right), \\
& |\mathscr{E}|=-1.3 \times 10^{-5}\left(\mathscr{E}_{I}=-1.5 \times 10^{-2}\right), \\
& \quad\left(\text { for } K n=0.05 \text { and }\left(r_{1}, r_{2}^{*}, r_{2}\right)=(0.199,0.416,0.597)\right), \\
& |\mathscr{M}|=7.8 \times 10^{-6}\left(\mathscr{M}_{I}=-2.1 \times 10^{-3}\right), \\
& \left|\mathscr{P}_{1}\right|=3.5 \times 10^{-6}\left(\mathscr{P}_{1 I}=-2.2 \times 10^{-1}\right), \\
& \left|\mathscr{P}_{2}\right|=1.4 \times 10^{-6}\left(\mathscr{P}_{2 I I I}=-2.1 \times 10^{-1}\right), \\
& |\mathscr{E}|=1.1 \times 10^{-5}\left(\mathscr{E}_{I}=-9.9 \times 10^{-3}\right), \\
& \quad\left(\text { for } \mathrm{Kn}=0.01 \text { and }\left(r_{1}, r_{2}^{*}, r_{2}\right)=(0.191,0.397,0.594)\right)
\end{aligned}
$$

\section{REFERENCES}

Aoki, K., Bardos, C., Dogbe, C. \& Golse, F. 2001a A note on the propagation of boundary induced discontinuities in kinetic theory. Math. Models Meth. Appl. Sci. 11 (9), 1581-1595.

Aoki, K., Degond, P. \& Mieussens, L. 2009 Numerical simulations of rarefied gases in curved channels: Thermal creep, circulating flow, and pumping effect. Commun. Comput. Phys. 6 (5), 919-954.

AoKi, K., Kanba, K. \& TAKatA, S. 1997 Numerical analysis of a supersonic rarefied gas flow past a flat plate. Phys. Fluids 9 (4), 1144-1161.

Aoki, K., Sone, Y. \& MAsukawa, N. 1995 A rarefied gas flow induced by a temperature field. In Rarefied Gas Dynamics (ed. J. Harvey \& G. Lord), pp. 35-41. Oxford University Press. 
Aoki, K., Sone, Y., Nishino, K. \& Sugimoto, H. 1991 Numerical analysis of unsteady motion of a rarefied gas caused by sudden changes of wall temperature with special interest in the propagation of a discontinuity in the velocity distribution function. In Rarefied Gas Dynamics (ed. A. E. Beylich), p. 222. VCH.

Aoki, K., Sone, Y. \& WANiguchi, Y. 1998 A rarefied gas flow induced by a temperature field: Numerical analysis of the flow between two coaxial elliptic cylinders with different uniform temperatures. Comput. Maths Applics. 35 (1/2), 15-28.

Aoki, K., Takata, S., Aikawa, H. \& Golse, F. $2001 b$ A rarefied gas flow caused by a discontinuous wall temperature. Phys. Fluids 13 (9), 2645-2661 and erratum: 13, 3843 (2001).

Bhatnagar, P. L., Gross, E. P. \& Krook, M. 1954 A model for collision processes in gases. I. Small amplitude processes in charged and neutral one-component systems. Phys. Rev. 94, 511-525.

BIRD, G. A. 1976 Molecular Gas Dynamics. Oxford University Press.

BIRD, G. A. 1994 Molecular Gas Dynamics and the Direct Simulation of Gas Flows. Oxford University Press.

Cercignani, C. 2000 Propagation phenomena in classical and relativistic rarefied gases. Transp. Theory Stat. Phys. 29, 607-614.

CHU, C. K. 1965 Kinetic-theoretic description of the formation of a shock wave. Phys. Fluids 8 (1), $12-22$.

Cornella, B. M., Ketsdever, A. D., Gimelshein, N. E. \& Gimelshein, S. F. 2011 Impact of separation distance on multi-vane radiometer configurations. In Rarefied Gas Dynamics (ed. D. A. Levin, I. J. Wysong \& A. L. Garcia), pp. 712-717. AIP.

EInstein, A. 1924 Zur Theorie der Radiometerkräfte. Z. Phys. 27, 1-6.

Galkin, V. S., Kogan, M. N. \& Fridlender, O. G. 1971 Free convection in a gas in the absence of external forces. Izv. Akad. Nauk SSSR Mekh. Zhidk. Gaza (3), 98-107.

GARciA, R. D. M. \& SiEwert, C. E. 2009 The linearized Boltzmann equation with Cercignani-Lampis boundary conditions: basic flow problems in a plane channel. Eur. J. Mech. B/Fluids 28, 387-396.

Gimelshein, N. E., Gimelshein, S. F., Ketsdever, A. D. \& Selden, N. P. $2011 a$ Shear force in radiometric flows. In Rarefied Gas Dynamics (ed. D. A. Levin, I. J. Wysong \& A. L. Garcia), pp. 661-666. AIP.

Gimelshein, S. F., Gimelshein, N. E., Ketsdever, A. D. \& Selden, N. P. $2011 b$ Analysis and applications of radiometeric forces in rarefied gas flows. In Rarefied Gas Dynamics (ed. D. A. Levin, I. J. Wysong \& A. L. Garcia), pp. 693-700. AIP.

Kennard, E. H. 1938 Kinetic Theory of Gases. McGraw-Hill.

KIM, C. 2011 Formation and propagation of discontinuity for Boltzmann equation in non-convex domains. Commun. Math. Phys. 308 (3), 641-701.

Kitamura, M., Tsutahara, M., Taguchi, S., Mitani, R., Enomura, M. \& Zhang, X. 2009 A study of promotion of sublimation phenomenon of freeze drying by using thermal edge flow. Trans. Japan Soc. Mech. Engrs B 75 (756), 1642-1648 (in Japanese).

Kogan, M. N., Galkin, V. S. \& Fridlender, O. G. 1976 Stresses produced in gases by temperature and concentration inhomogeneities. New type of free convection. Sov. Phys. Usp. 19, 420-428.

Loeb, L. B. 1961 Kinetic Theory of Gases, 3rd edn. Dover.

MAXWELl, J. C. 1879 On stresses in rarified gases arising from inequalities of temperature. Phil. Trans. R. Soc. Lond. 170, 231-256.

OHwAdA, T., SONE, Y. \& AOKI, K. $1989 a$ Numerical analysis of the shear and thermal creep flows of a rarefied gas over a plane wall on the basis of the linearized Boltzmann equation for hard-sphere molecules. Phys. Fluids A 1 (9), 1588-1599.

OhwadA, T., Sone, Y. \& AOKI, K. $1989 \mathrm{~b}$ Numerical analysis of the Poiseuille and thermal transpiration flows between two parallel plates on the basis of the Boltzmann equation for hard-sphere molecules. Phys. Fluids A 1 (12), 2042-2049 and erratum: 2, 639 (1990).

OTA, M., NAKAO, T. \& SAKAмото, M. 2001 Numerical simulation of molecular motion around laser microengine blades. Math. Comput. Simul. 55, 223-230. 


\section{Rarefied gas flow around a sharp edge}

REYNOLDS, O. 1876 On the forces caused by the communication of heat between a surface and a gas; and on a new photometer. Phil. Trans. R. Soc. Lond. 166, 725-735.

Scandurra, M., Iacopetti, F. \& Colona, P. 2007 Gas kinetic forces on thin plates in the presence of thermal gradients. Phys. Rev. E 75, 026308-5.

Selden, N., Ngalande, C., Gimelshein, N., Gimelshein, S. \& Ketsdever, A. $2009 a$ Origins of radiometric forces on a circular vane with a temperature gradient. J. Fluid Mech. 634, 419-431.

Selden, N., Ngalande, C., Gimelshein, S., Muntz, E. P., Alexeenko, A. \& Ketsdever, A. $2009 b$ Area and edge effects in radiometric forces. Phys. Rev. E 79, 041201.

Sharipov, F. \& Seleznev, V. 1998 Data on internal rarefied gas flows. J. Phys. Chem. Ref. Data 27 (3), 657-706.

Sone, Y. 1966 Thermal creep in rarefied gas. J. Phys. Soc. Japan 21, 1836-1837.

SONE, Y. 1969 Asymptotic theory of flow of rarefied gas over a smooth boundary I. In Rarefied Gas Dynamics (ed. L. Trilling \& H. Y. Wachman), vol. 1. pp. 243-253. Academic Press.

Sone, Y. 1971 Asymptotic theory of flow of rarefied gas over a smooth boundary II. In Rarefied Gas Dynamics (ed. D. Dini), vol. 2. pp. 737-749. Editrice Tecnico Scientfica.

Sone, Y. 1973 New kind of boundary layer over a convex solid boundary in a rarefied gas. Phys. Fluids 16 (9), 1422-1424.

SONE, Y. 1984 Highly rarefied gas around a group of bodies with various temperature distributions i small temperature variation. J. Méc. Théor. Appl. 3, 315-328.

SONE, Y. 1985 Highly rarefied gas around a group of bodies with various temperature distributions ii arbitrary temperature variation. J. Méc. Théor. Appl. 4, 1-14.

Sone, Y. 1991 Asymptotic theory of a steady flow of a rarefied gas past bodies for small Knudsen numbers. In Advances in Kinetic Theory and Continuum Mechanics (ed. R. Gatignol \& Soubbaramayer), pp. 19-31. Springer.

SONE, Y. 2000 Flows induced by temperature fields in a rarefied gas and their ghost effect on the behaviour of a gas in the continuum limit. Annu. Rev. Fluid Mech. 32, 779-811.

Sone, Y. 2002 Kinetic Theory and Fluid Dynamics. Birkhäuser, Supplementary Notes and Errata: Kyoto University Research Information Repository (http://hdl.handle.net/2433/66099).

Sone, Y. 2007 Molecular Gas Dynamics: Theory, Techniques, and Applications. Birkhäuser, Supplementary Notes and Errata: Kyoto University Research Information Repository (http://hdl.handle.net/2433/66098).

Sone, Y., Aoki, K., Takata, S., Sugimoto, H. \& Bobylev, A. V. 1996 Inappropriateness of the heat-conduction equation for description of a temperature field of a stationary gas in the continuum limit: examination by asymptotic analysis and numerical computation of the Boltzmann equation. Phys. Fluids 8, 628-638 and erratum: 8, 841.

Sone, Y \& Sugimoto, H 1990 Strong evapouration from a plane condensed phase. In Adiabatic Waves in Liquid-Vapor Systems (ed. G. E. A. Meier \& P. A. Thompson), pp. 293-304. Springer.

Sone, Y. \& Sugimoto, H. 1993 Kinetic theory analysis of steady evapourating flows from a spherical condensed phase into a vacuum. Phys. Fluids A 5, 1491-1511.

Sone, Y. \& TAKATA, S. 1992 Discontinuity of the velocity distribution function in a rarefied gas around a convex body and the $\mathrm{S}$ layer at the bottom of the Knudsen layer. Transp. Theory Stat. Phys. 21, 501-530.

SONE, Y. \& TANAKA, S. 1980 Thermal stress slip flow induced in rarefied gas between noncoaxial circular cylinders. In Theoretical and Applied Mechanics (ed. F. P. J. Rimrott \& B. Tabarrok), pp. 405-416. North-Holland.

Sone, Y. \& Yoshimoto, M. 1997 Demonstration of a rarefied gas flow induced near the edge of a uniformly heated plate. Phys. Fluids 9, 3530-3534.

Sugimoto, H. 2009 Experiment on the gas separation effect of the pump driven by the thermal edge flow. In Rarefied Gas Dynamics (ed. T. Abe), pp. 1123-1128. AIP.

Sugimoto, H. \& Sone, Y. 1992 Numerical analysis of steady flows of a gas evapourating from its cylindrical condensed phase on the basis of kinetic theory. Phys. Fluids A 4, 419-440.

Sugimoto, H. \& Sone, Y. 2005 Vacuum pump without a moving part by thermal edge flow. In Rarefied Gas Dynamics (ed. M. Capitelli), pp. 168-173. AIP. 
TAGUCHI, S. \& AOKI, K. 2011 Numerical analysis of rarefied gas flow induced around a flat plate with a single heated side. In Rarefied Gas Dynamics (ed. D. A. Levin, I. J. Wysong \& A. L. Garcia), pp. 790-795. AIP.

Takata, S., Funagane, H. \& AOKI, K. 2010 Fluid modelling for the Knudsen compressor: case of polyatomic gases. Kinet. Relat. Mod. 3 (2), 353-372.

TAKatA, S., Sone, Y. \& AOKI, K. 1993 Numerical analysis of a uniform flow of a rarefied gas past a sphere on the basis of the Boltzmann equation for hard-sphere molecules. Phys. Fluids A 5, 716-737.

Wadsworth, D. C. \& Muntz, E. P. 1996 A computational study of radiometric phenomena for powering microactuators with unlimited displacements and large available forces. J. Microelectromech. Syst. 5 (1), 59-65.

Welander, P. 1954 On the temperature jump in a rarefied gas. Ark. Fys. 7, 507. 